\title{
Critical Dynamics and Coupling in Bursts of Cortical Rhythms Indicate Non-Homeostatic Mechanism for Sleep-Stage Transitions and Dual Role of VLPO Neurons in Both Sleep and Wake
}

\author{
๑Dabrizio Lombardi, ${ }^{1,4}$ (D) Manuel Gómez-Extremera, ${ }^{2}$ Pedro Bernaola-Galván, ${ }^{2,3}$ @Ramalingam Vetrivelan, ${ }^{5}$ \\ DClifford B. Saper, 5 (DThomas E. Scammell, ${ }^{5}$ and DPlamen Ch. Ivanov ${ }^{1,6}$ \\ ${ }^{1}$ Keck Laboratory for Network Physiology, Department of Physics, Boston University, Boston, Massachusetts 02215, ${ }^{2}$ Departamento de Física Aplicada II, \\ ETSI de Telecomunicación, Universidad de Málaga, Málaga 29071, Spain, ${ }^{3}$ Institute Carlos I for Theoretical and Computational Physics, 29071 Málaga, \\ Spain, ${ }^{4}$ Institute of Science and Technology Austria, A-3400 Klosterneuburg, Austria, ${ }^{5}$ Department of Neurology, Beth Israel Deaconess Medical Center and \\ Harvard Medical School, Boston, Massachusetts 02215, and ${ }^{6}$ Harvard Medical School and Division of Sleep Medicine, Brigham and Women Hospital, \\ Boston, Massachusetts 02215
}

Origin and functions of intermittent transitions among sleep stages, including brief awakenings and arousals, constitute a challenge to the current homeostatic framework for sleep regulation, focusing on factors modulating sleep over large time scales. Here we propose that the complex micro-architecture characterizing sleep on scales of seconds and minutes results from intrinsic non-equilibrium critical dynamics. We investigate $\theta$ - and $\delta$-wave dynamics in control rats and in rats where the sleep-promoting ventrolateral preoptic nucleus (VLPO) is lesioned (male Sprague-Dawley rats). We demonstrate that bursts in $\theta$ and $\delta$ cortical rhythms exhibit complex temporal organization, with long-range correlations and robust duality of power-law ( $\theta$-bursts, active phase) and exponential-like $(\delta$-bursts, quiescent phase) duration distributions, features typical of non-equilibrium systems self-organizing at criticality. We show that such non-equilibrium behavior relates to anti-correlated coupling between $\theta$ - and $\delta$-bursts, persists across a range of time scales, and is independent of the dominant physiologic state; indications of a basic principle in sleep regulation. Further, we find that VLPO lesions lead to a modulation of cortical dynamics resulting in altered dynamical parameters of $\theta$ - and $\delta$-bursts and significant reduction in $\theta-\delta$ coupling. Our empirical findings and model simulations demonstrate that $\theta-\delta$ coupling is essential for the emerging nonequilibrium critical dynamics observed across the sleep-wake cycle, and indicate that VLPO neurons may have dual role for both sleep and arousal/brief wake activation. The uncovered critical behavior in sleep- and wake-related cortical rhythms indicates a mechanism essential for the micro-architecture of spontaneous sleep-stage and arousal transitions within a novel, nonhomeostatic paradigm of sleep regulation.

Key words: brain rhythms; cortical dynamics; criticality; sleep regulation; VLPO

Significance Statement

We show that the complex micro-architecture of sleep-stage/arousal transitions arises from intrinsic non-equilibrium critical dynamics, connecting the temporal organization of dominant cortical rhythms with empirical observations across scales. We link such behavior to sleep-promoting neuronal population, and demonstrate that VLPO lesion (model of insomnia) alters dynamical features of $\theta$ and $\delta$ rhythms, and leads to significant reduction in $\theta-\delta$ coupling. This indicates that VLPO neurons may have dual role for both sleep and arousal/brief wake control. The reported empirical findings and modeling simulations constitute first evidences of a neurophysiological fingerprint of self-organization and criticality in sleep- and wake-related cortical rhythms; a mechanism essential for spontaneous sleep-stage and arousal transitions that lays the bases for a novel, non-homeostatic paradigm of sleep regulation. 


\section{Introduction}

Sleep periods exhibit numerous transitions among sleep stages and short awakenings, with intermittent fluctuations within sleep stages that trigger micro-states and brief arousals (Halász, 1998; Hirshkowitz, 2002; Lo et al., 2002). Such behavior is typically observed in non-equilibrium systems characterized by multicomponent nonlinear feedback interactions and exhibiting critical behavior, with irregular alternation between active and quiescent phases (Bak, 1996; Chialvo, 2010; Munoz, 2018). This constitutes a challenge to the current homeostatic framework for sleep regulation, which considers sleep as an equilibrium process, and focuses on factors modulating sleep over large time scales, such as homeostatic sleep drive, sleep propensity and inertia, and ultradian and circadian rhythms (Borbély and Achermann, 1999; Saper et al., 2005; Brown et al., 2012). Existing homeostatic models, although successfully providing a good description of consolidated sleep and wakefulness over time scales of hours (Borbély and Achermann, 1999; Achermann and Borbély, 2003; Saper et al., 2001, 2010), do not capture the emergent complexity of transient and abrupt behaviors at scales of seconds and minutes (Lo et al., 2004; Olbrich et al., 2011), and do not account for the related dynamics of bursts in cortical rhythms.

The intrinsic fluctuations in cortical rhythmic activity in response to feedback interactions among sleep- and wake-promoting neuronal groups, and the corresponding complex temporal patterns of intermittent transitions in sleep micro-architecture, suggest that critical dynamics may underlie sleep regulation at small time scales, in parallel with the well established homeostatic behavior at large time scales. Such hypothesis is further motivated by recent work showing a peculiar coexistence of power-law and exponential probability distributions for the durations of brief awakenings/arousals and sleep bouts, in both humans and animal models (Lo et al., 2002, 2004; Blumberg et al., 2005; Behn et al., 2007; Dvir et al., 2018). This scenario is closely reminiscent of non-equilibrium systems self-tuning at criticality, where a quiescent phase with exponential dynamics coexists with an active phase characterized by bursts/avalanches with power-law distributed sizes and durations (Boffetta et al., 1999; Chialvo, 2010; Munoz, 2018). Because brief awakenings/arousals can be viewed as "active" states of the brain that interrupt the "inactive" phase represented by sleep periods, the coexistence of power-law distributed arousal durations with sleep bouts durations following exponential behavior has been interpreted as a fingerprint of criticality in sleep dynamics (Lo et al., 2004, 2013).

To test this hypothesis, we investigate the dynamics of dominant brain waves in the sleep-wake cycle of rats in relation to the neuronal circuitry responsible for wake and sleep control. In particular, we focus on the ventrolateral preoptic nucleus (VLPO), a key sleep promoting brain region (Sherin et al., 1996), and study whether alterations in the sleep-wake cycle are mirrored by a reorganization of dominant brain rhythms. The sleep-wake cycle of rats is largely dominated by the $\delta$ and $\theta$ rhythm. During

Received June 1, 2019; revised Sept. 7, 2019; accepted Oct. 7, 2019.

Author contributions: F.L. and P.C.I. designed research; F.L, M.G.-E., P.B.-G., R.V., C.B.S., T.E.S., and P.C.I. performed research; M.G.-E. and P.B.-G. analyzed data; F.L. and P.C.I. wrote the paper.

This work was supported by the National Institutes of Health (Grant 1R01-HL098437), the US-Israel Binational Science Foundation (Grant 2012219), the Office of Naval Research (Grant 000141010078), the Keck Foundation, the Spanish Junta de Andalucia (FQM-7964), the Marie Sklodowska-Curie Grant (754411), NIH-NIA (AG09975) and the NIH-NHLBI (HL095461).

The authors declare no competing financial interests.

Correspondence should be addressed to Plamen Ch. Ivanov at plamen@buphy.bu.edu.

https://doi.org/10.1523/JNEUROSCI.1278-19.2019

Copyright $\odot 2020$ the authors non-rapid eye movement sleep (NREM) sleep, cortical activity is characterized by $\delta$ rhythm, low-frequency high-amplitude oscillations referred to as slow-wave activity (Steriade et al., 1993), whereas REM sleep and arousals/wake state are characterized by $\theta$ rhythm, desynchronized and localized oscillations of higher frequency and lower amplitude (Brown et al., 2012). Lesions of the central cluster of the VLPO lead to a large decrease in delta power and NREM sleep time, and a fragmentation of the sleep-wake cycle (Lu et al., 2000). REM sleep may also be affected by lesions in the VLPO area, especially in the medial and dorsal extended VLPO region (Vetrivelan et al., 2012). However, the influence of VLPO neurons on the dynamics and temporal organization of $\delta$ and $\theta$ waves during the sleep-wake cycle has not been investigated.

To this aim, we analyze long-term continuous EEG recordings in control rats and rats with lesions in the VLPO, and dissect emergent signatures of criticality in the dynamics of $\theta$ - and $\delta$-bursts in relation to VLPO neuronal integrity. We link alterations of the sleep-wake cycle with non-equilibrium properties of the underlying dynamics, providing first evidence for a criticality-based framework of sleep regulation that unifies sleep stage and arousal transitions with basic dynamics of dominant cortical rhythms.

\section{Materials and Methods}

\section{Experimental setup and statistical analysis}

Twelve pathogen-free, 12- to 16-week-old male Sprague-Dawley rats (300-365 g; Harlan) were used for this study. Data analyzed here were selected from a previously presented study (Vetrivelan et al., 2012) with 7 control and 34 VLPO-lesioned rats. The VLPO-lesioned group used for the present study includes all animals with $>85 \%$ cell loss in the VLPO; $n=7$ of the entire cohort of 34 VLPO-lesioned rats. One control rat and one VLPO-lesioned rat were excluded from the final analysis after the preprocessing procedure. For the purpose of our study of sleep microdynamics, we selected and analyzed rats with $<10 \%$ artifacts in the EEG (see Data preprocessing). The experimental procedure is briefly summarized below. For further details regarding data collection and protocol, please refer to Vetrivelan et al. (2012).

Rat surgery. Animals were anesthetized with chloral hydrate (350 $\mathrm{mg} / \mathrm{kg}$ body weight, i.p.) and the fur over the skull was shaved, and the skin was prepped with betadine and alcohol. The animals were then fixed in a stereotaxic frame. All surgery was conducted using sterile autoclaved instruments and under aseptic conditions. To produce VLPO lesions, orexin-B-saporin (OX-SAP; Advanced Targeting Systems) was injected into the VLPO as described below. Skin $(\sim 1 \mathrm{~cm})$ was incised and small burr holes were drilled in the skull corresponding to the VLPO coordinates. A glass micropipette (tip diameter $10-20 \mu \mathrm{m}$ ) was then lowered to coordinates (AP: -0.6 from bregma, L: $1.00, \mathrm{DV}: 8.5$ ) corresponding to the VLPO as per the rat atlas of Paxinos and Watson (2004) and OX-SAP (200 $\mathrm{nl}$ of $0.1 \%$ solution) was injected using a compressed air delivery system (Amaral and Price, 1983). The micropipette was then left in place for $5 \mathrm{~min}$ and slowly withdrawn. Control animals received saline (vehicle) injections into the VLPO. Following the injections, four EEG screw electrodes were implanted into the skull, in the frontal (2) and parietal bones (2) of each side, and two flexible EMG wire electrodes were placed into the neck muscles for the collection of sleep-wake data. Four burr holes were made in the skull ( $1 \mathrm{~mm}$ rostral and $3 \mathrm{~mm}$ lateral; $3 \mathrm{~mm}$ caudal and $3 \mathrm{~mm}$ lateral) and EEG electrodes were inserted into those burr holes so that they would be close to dura mater. EMG wire electrodes were placed onto the neck extensor muscles on either side. The free ends of the leads were soldered into a socket that was attached to the skull with dental cement, and the incision was then closed by wound clips (Lu et al., 2000, 2002).

EEG recording across dark and light and sleep-wake analysis. EEG recordings from each rat were performed on Day 20 post-lesion using Grass polygraph. The rats were connected via flexible recording cables to a commutator, which in turn was connected to a Grass polygraph and 
computer. The rats were habituated to the connecting cables and the recording room conditions for $2 \mathrm{~d}$ and then uninterrupted recordings of the EEG/EMG (sampling rate $256 \mathrm{~Hz}$ ) and time-lock video were conducted for $48 \mathrm{~h}$ ( $2 \mathrm{~d}, 12 \mathrm{~h}$ light/dark) beginning of Day 20 postlesion. EEG signals were recorded continuously from the frontal and parietal electrode on both left and right hemisphere. The parietal electrode picks up $\theta$ rhythm from the hippocampus during REM sleep; both the frontal and parietal electrodes pick up $\theta$ rhythm during wakefulness. Hippocampal $\theta$ rhythm can be also present during wakefulness, mainly during locomotion and cognitive wakefulness. The signal analyzed in this study is the difference between frontal and parietal EEG electrode potentials (frontal-parietal EEG) from one hemisphere (ipsilateral). The EEG/EMG data of each rat was divided into $12 \mathrm{~s}$ epochs and visually scored as wake, NREM sleep, or rapid eye movement (REM) sleep using scoring criteria previously described (Lu et al., 2000). Wakefulness was identified by the presence of desynchronized-EEG and high-EMG activity. NREM sleep was identified by the presence of a high-amplitude, slow-wave EEG and low-EMG activity relative to that of waking. REM sleep was identified by the presence of regular theta activity on EEG, coupled with low-EMG activity relative to that of NREM sleep. When two states (for example, NREM sleep and wake) occurred within a $12 \mathrm{~s}$ epoch, the epoch was scored for the state that predominated. Scoring was done before histological examination, so the scorer was unaware of the extent of the lesions. The daily percentage of time spent in wake, NREM sleep, and REM sleep and frequency and durations of episodes of each stage were calculated.

Histology. On completion of the recordings, the rats were deeply anesthetized (chloral hydrate, $500 \mathrm{mg} / \mathrm{kg}$ ) and transcardially perfused with $100 \mathrm{ml}$ saline, followed by $500 \mathrm{ml}$ of neutral phosphate buffered formalin (ThermoFisher Scientific). The brains were removed and processed for Nissl staining. For this, the harvested brains were sectioned in the coronal plane on a freezing microtome into four series of $40 \mu \mathrm{m}$ sections and one series was mounted on gelatin-coated slides, washed in $\mathrm{H}_{2} \mathrm{O}$, and washed in PBS. Sections were then incubated in $0.25 \%$ thionin in $0.1 \mathrm{M}$ acetate buffer solution for $2 \mathrm{~min}$, differentiated in graded ethanols, delipidated in xylene and coverslipped (Lu et al., 2000).

Statistical analysis. Power-law exponent and fitting parameters for burst duration distributions were evaluated for each rat and condition ( $24 \mathrm{~h}: 12 \mathrm{~h}$ dark/light periods) in both the control group and VLPO. Pairwise comparisons between groups and conditions were conducted (see Figs. 2 and 3). The temporal correlations detrended fluctuation analysis (DFA) exponent (see Fig. 11) and $\theta-\delta$ coupling Spearman's correlation coefficient (see Fig. 12) were evaluated for each rat in both groups, and then pairwise comparisons between conditions and groups were conducted. Individual group data were tested for normality using the Shapiro-Wilk test. Pairwise comparisons were conducted using Students two-tailed $t$ test with Welch's correction, unless the Shapiro-Wilk test was significant, in which case the nonparametric Mann-Whitney $U$ test was used. Paired tests: control $12 \mathrm{~h}$ dark versus $12 \mathrm{~h}$ light period; VLPOlesioned $12 \mathrm{~h}$ dark versus $12 \mathrm{~h}$ light period. Comparisons between control and VLPO-lesioned group were performed for each condition $(12 \mathrm{~h}$ dark/light period), and for the $24 \mathrm{~h}$ period. Surrogate tests were used to test significance of correlations between theta- and delta-bursts, and are described in Data analysis. All statistical tests were performed in MATLAB (MathWorks).

\section{Data preprocessing}

EEG recordings were first normalized to zero mean, $\mu=0$, and unit SD, $\sigma=1$. For each rat, EEG signals were visually inspected and noisy segments were discarded according to the following semiautomatic procedure. Data were first examined to identify most typical noise/artifact waveforms and their specific characteristics, such as amplitude and average duration $\langle T\rangle$ expressed in number of sampling points. Based on this preliminary analysis, two amplitude thresholds $S_{1}$ and $s_{2}$, with $S_{1}>s_{2}$, were introduced. The values of these thresholds are multiples of the EEG signal SD $\sigma$, and depend on the specific noise/artifact waveform for each particular rat. Typical values of threshold $S_{1}$ range between $3.5 \sigma$ and $6 \sigma$, and $s_{2}$ is between $2.5 \sigma$ and $4 \sigma$. EEG signals were then scanned using a non-overlapping window $W_{1}=\langle T\rangle / 2$. Whenever a window $W_{1}$ contained a significant number of points (e.g., $n_{s}>15$; EEG signals were sampled at $256 \mathrm{~Hz}$ ) with amplitude exceeding the threshold $S_{1}$, our algorithm identifies an artifact, and a non-overlapping sub-window of size $w_{2}<W_{1}$ was used to scan again the identified artifact segment and clean it up. Specifically, this second step works as follows: inside the artifact window $W_{1}$, segments of length $w_{2}$ containing points with amplitude exceeding the threshold $s_{2}$ are sequentially cleaned up by substituting all points in $w_{2}$ with zeros. An artifact segment $w_{2}$ that follows a preceding cleaned segment but does not contain points exceeding the threshold $s_{2}$ is also cleaned; this removes subthreshold points in $W_{1}$ that belong to the decaying part of an artifact. In this case, such procedure continues and the following sub-windows $w_{2}$ are also cleaned until the signal crosses zero. These steps are repeated until the entire artifact segment $W_{1}$ is scanned. Finally, 500 points $(\approx 2 \mathrm{~s})$ are removed from the EEG on both sides of the cleaned artifact segment $W_{1}$, to eliminate possible pre-artifact and post-artifact influence on the signal. These steps carefully take into account the general slow decaying waveform of some EEG artifacts, and are needed to ensure an optimal cleaning of the data. For all rats in this study $W_{1}=500$ and $w_{2}=100$ data points. At the end of the preprocessing procedure EEG signals were visually inspected to ensure that all noisy segments were properly removed. The total length of removed noisy segments ranges between 5 and $10 \%$ of the $48 \mathrm{~h}$ recording for each rat. Of the 7 control and 7 VLPO-lesioned rats, 1 control and 1 VLPO rat had $>10 \%$ of the signal removed after the preprocessing, and correspondingly were removed from the analysis. The code used for EEG preprocessing is available upon request.

Data filtering. Data were bandpass filtered in the range $0.5-25 \mathrm{~Hz}$ using a FIR (finite impulse response) filter designed in MATLAB.

\section{Data analysis}

Spectral analysis. The clean EEG signal is divided in $N$ non-overlapping windows of size $w$ and the spectral power in the $\delta$ band $(0.5-4 \mathrm{~Hz}), S_{\delta}$, and in the $\theta$ band $(4-8 \mathrm{~Hz}), S_{\theta}$, is estimated in each window using Welch's method (Welch, 1967). The analysis is performed for several values of the window size $w$, from 2 to $10 \mathrm{~s}$. Results are generally independent of $w$, as shown in Figures 5, 6, and 7, and extensively discussed in the main text.

$\theta$ and $\delta$ burst detection and definition. The ratio $R_{\theta \delta}=S_{\theta} / S_{\delta}$ between $\theta$ and $\delta$ power is calculated in each window $k$, with $k=1,2, \ldots, N$, and a time series $R_{\theta \delta}(k)$ is obtained. Given a threshold $T h \geq 1$, a $\theta$-burst is defined as a sequence of $n$ consecutive windows where $R_{\theta \delta}>$ Th, while a $\delta$-burst consists in a sequence of $n$ consecutive windows where $R_{\theta \delta}<1 /$ Th (Fig. 1). The duration of a burst is given by $d=n \cdot w$. Durations of $\theta(\delta)$ bursts are denoted by $d_{\theta}\left(d_{\delta}\right)$. The threshold Th is set equal to 1 throughout the analysis. Results are independent of $T h$, as shown in Figures 4, 5, and 6 , and extensively discussed in the main text.

Surrogate test for $\theta$ - and $\delta$-burst duration distributions. For each rat, the time series $R_{\theta \delta}(k)$ is randomly reshuffled to obtain a surrogate $R_{\theta \delta}^{*}\left(k^{\prime}\right)$. Surrogate $\theta$ - and $\delta$-bursts durations (see Fig. 2) are then calculated from $R_{\theta \delta}^{*}\left(k^{\prime}\right)$ following the procedure illustrated in the previous paragraph. The corresponding $\theta$ - and $\delta$-burst duration distributions are shown in Figure 2, insets, together with distributions from original data.

Definition of quiet time $\Delta t$. A quiet time $\Delta t$ is defined as the time interval between the ending time of a burst $t_{j}^{e}$ and the starting time $t_{j+1}^{s}$ of the following one, namely $\Delta t_{j}=t_{j+1}^{s}-t_{j}^{e}$.

Data binning. Probability distributions of $\theta$-burst durations are calculated using logarithmic binning, i.e., linear binning in logarithmic scale. Denoting a set of bin boundaries as $B=\left(b_{1}, b_{2}, \ldots, b_{k}\right)$ and fixing $b_{1}=$ $0.03 w$, the logarithmic bins fulfill the relation $b_{i+1}=b_{i} \cdot 10^{c}$, which implies that the bin size is constant in logarithmic scale, i.e., $\log b_{i+1}-\log b_{i}=c$. The following bin size $c$ have been used in this study: Figures 2-9 and 15; $c=0.18$. Probability distributions of $\delta$-burst durations are calculated using the following binning procedure. Given a window size $w$, the bin boundaries $e_{1}, e_{2}, \ldots, e_{n}, \ldots, e_{k}$ are obtained using the recursive relation $e_{n}=e_{1}+w \sum_{i=2}^{n} b^{i-1}$, with $e_{1}=0.5 w$ and $n \geq 2$. The following values of the parameter $b$ have been used in this study: Figures 10, $b=1.6$; all other figures, $b=1.2$.

Duration distributions: error bars. An error $\delta \mathrm{P}$ is associated to each bin of the distribution for pooled data presented in Figures 2 and 3. The 
number of data $n$ in a particular interval $[D, D$ $+d D]$ can be considered as given by the binomial distribution, given that the total number of data $N$ is much larger than the range of correlations. Therefore, the associated $\mathrm{SD}$ is $\sigma=\sqrt{N p(1-p)}$. Because $P(D)=n /(N d D)$, the error on $P(D)$ is given by the following:

$$
\delta P=\frac{1}{d D} \sqrt{\frac{p(1-p)}{N}},
$$

where $p=P(D) d D$ is the probability to observe a duration $D$ in the range $[D, D+d D]$ and $N$ is the total number of bursts and $d D$ is the corresponding bin size. Power-law fits for $\theta$-burst duration distributions shown in Figures 2 and 3 are performed on the pooled data. Estimates are reported in the corresponding figure captions together with associated errors on the fit.

Spearman's correlation. Given to variables $X$ and $Y$, the Spearman's correlation coefficient is defined as follows:

$$
\rho_{s}=\frac{\operatorname{cov}\left(r g_{X}, r g_{Y}\right)}{\sigma_{r g X} \sigma_{r g_{Y}}},
$$

where $r g_{X}$ and $r g_{Y}$ are the tied rankings of $X$ and $Y$, respectively, $\sigma_{r g X}$ and $\sigma_{r g Y}$ their SDs, and cov $\left(r g_{X}, r g_{Y}\right)$ indicates the covariance between $r g_{X}$ and $r g_{Y}$.

Surrogate test for correlations between consecutive $\theta$ - and $\delta$-burst duration. To test significance of correlations between consecutive $\theta$ and $\delta$-burst durations, a surrogate sequence of burst durations is generated for each rat by randomly reshuffling the original order of $\theta$ - and $\delta$-bursts. The Spearman's correlation coefficient $\rho_{\mathrm{s}}$ between consecutive $\theta$ - and $\delta$-bursts is calculated for each surrogate. The average Spearman's correlation coefficient obtained from all surrogates is then compared with the average correlation coefficient calculated from the original sequences of burst durations via $t$ test (see Results; Fig. 12). Correlation coefficients for surrogate data for both control and VLPO-lesioned rats during dark, light, and $24 \mathrm{~h}$ are all with value $\left|\rho_{s}\right|<10^{3}$.

DFA. The DFA is a method based on random walk (Peng et al., 1994). It improves the classical fluctuation analysis (FA) for nonstationary signals where embedded polynomial trends mask the intrinsic correlation properties in the fluctuations (Peng et al., 1994). The performance of DFA for signals with different types of non-stationarities and artifacts has been extensively studied and compared with other methods of correlation analysis (Taqqu et al., 1995; Hu et al., 2001; Chen et al., 2005; Xu et al., 2005). The DFA method is briefly described by the following steps (Peng et al., 1994):

(1) A given signal $u_{i}(i=1, \ldots, N$, where $N$ is the length of the signal) is integrated to obtain $y(k) \equiv \sum_{i=1}^{k}[u(i)-\langle u\rangle]$, where $\langle u\rangle$ is the mean of $u_{i}$;

(2) The integrated signal $y(k)$ is divided into boxes of equal length $n$;

(3) In each box of length $n$ we fit $y(k)$ using a first-order polynomial function, which represents the trend in that box. The $y$-coordinate of the fit curve in each box is denoted by $y_{n}(k)$;

(4) The integrated profile $y(k)$ is detrended by subtracting the local trend $y_{n}(k)$ in each box of length $n$ :
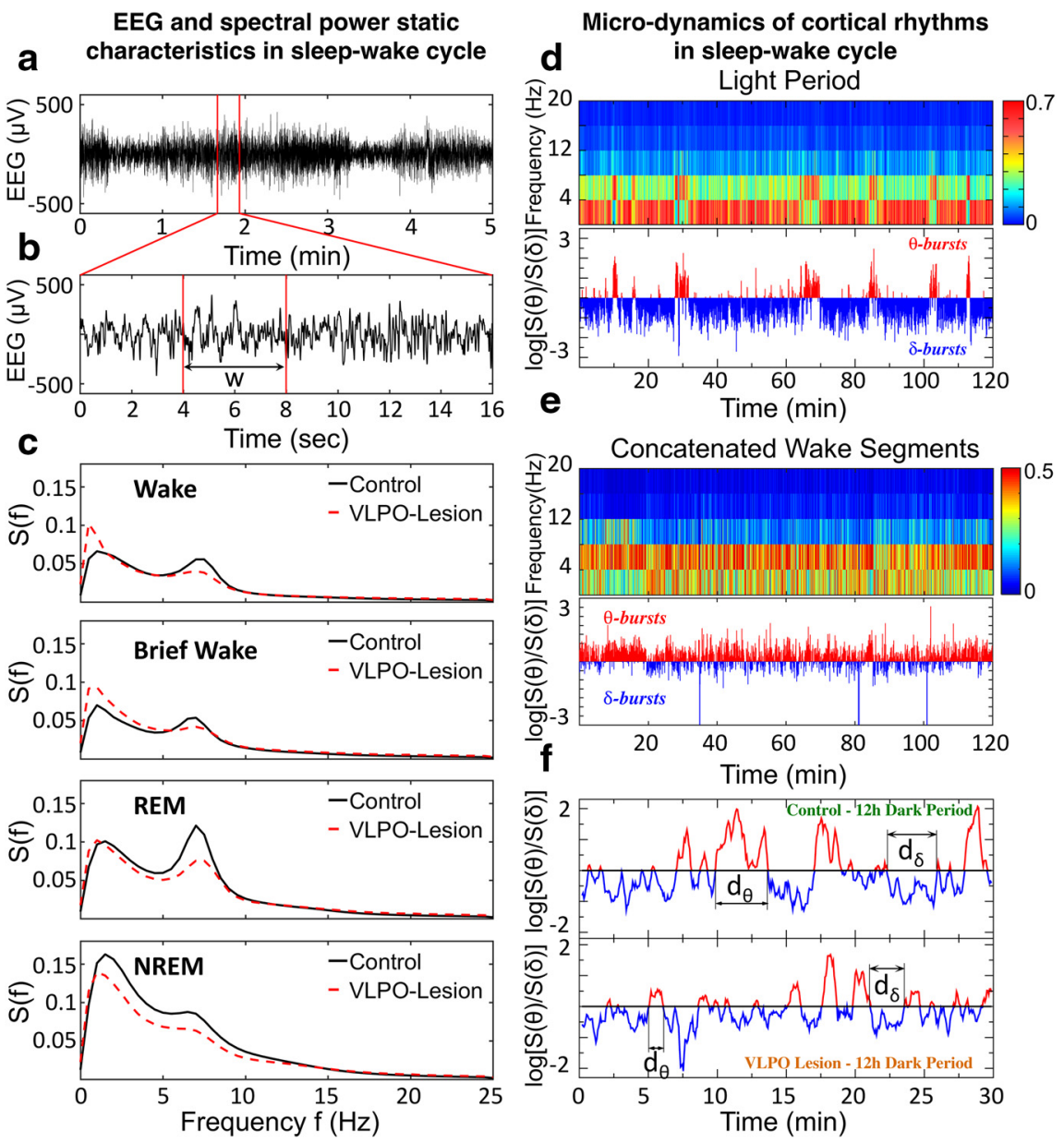

Figure 1. Cortical activity across the sleep-wake cycle is characterized by intermittent transitions between distinct dominant brain rhythms. $\boldsymbol{a}$, Representative $5 \mathrm{~min}$ EEG trace for a control rat during a $12 \mathrm{~h}$ dark period. $\boldsymbol{b}, \mathrm{A} 16 \mathrm{~s}$ segment from the $5 \mathrm{~min}$ EEG trace shown in $\boldsymbol{a}$. The time evolution of the EEG signal is analyzed by evaluating the spectral power in several frequency bands on non-overlapping windows of length $w$, as shown in $\boldsymbol{d}-\boldsymbol{f}$. c, Average power spectra for control and VLPO-lesioned rats during Wake, brief wake periods ( $<1 \mathrm{~min}$ ), REM, and NREM sleep. $\boldsymbol{d}$, Top, Spectrogram obtained from cortical EEG signal of a control rat over a $2 \mathrm{~h}$ segment of $12 \mathrm{~h}$ lights-on period. Spectral power is calculated in non-overlapping time windows $w=4 \mathrm{~s}$, and is color coded over a range $(0-20 \mathrm{~Hz})$ of physiologically-relevant frequencies. Segments in red indicate bursts of prominent activity in the low-frequency band ( $0-4 \mathrm{~Hz}$, corresponding to $\delta$ waves) and in the intermediate frequency band ( $4-8 \mathrm{~Hz}$, corresponding to $\theta$ waves). Bottom, Ratio $R_{\theta \delta}=S(\theta) / S(\delta)$ of the spectral power in the $\theta$ and $\delta$ band in logarithmic scale obtained from the spectrogram at the top. Values $R_{\theta \delta}$ above $\log (T h)=0(T h=1)$ indicate predominance of $\theta$ rhythm (red), whereas values below $\log (T h)=0$ correspond to predominance of $\delta$ rhythm (blue). $e$, Top, Spectrogram obtained from cortical EEG signal over $2 \mathrm{~h}$ of concatenated wake segments. Spectral power is calculated in non-overlapping time windows $w=4 \mathrm{~s}$, and is color coded as in Ratio $R_{\theta \delta}$ of the spectral power in $\theta$ and $\delta$ band in logarithmic scale obtained from the spectrogram at the top. Values $R_{\theta \delta}$ above $\log (\mathrm{Th})=0(\mathrm{Th}=1)$ indicate predominance of $\theta$ rhythm $(\mathrm{red})$, whereas values below $\log (\mathrm{Th})=0$ correspond to predominance in the $\theta$ band. $f$, Smoothed ratio $R_{\theta \delta}$ of the spectral power in the $\theta$ and $\delta$ band during 30 min segment of $12 \mathrm{~h}$ dark (lights-off period for a control rat (top) and a VLP0-lesioned rat (bottom). $R_{\theta \delta}$ is calculated on non-overlapping windows $w=4 \mathrm{~s}$ and the smoothing is performed using a 5 point moving average. $\theta$-and $\delta$-bursts are defined as sequences of consecutive windows where either the power in $\theta$ or $\delta$ band is dominant.

$$
Y(k) \equiv y(k)-y_{n}(k)
$$

(5) For a given box length $n$, calculate the root mean square (rms) fluctuation function for this integrated and detrended signal:

$$
F(n) \equiv \sqrt{\frac{1}{N} \sum_{k=1}^{N}[Y(k)]^{2}} ;
$$

(6) Repeat the above computation over a broad range of box lengths $n$, where $n$ represents a specific space or time scale, to obtain a functional relationship between $F(n)$ and $n$. 
For a power-law correlated time series, the average rms fluctuation function $F(n)$ and the box size $n$ are connected by a power-law relation, that is $F(n) \sim n^{\alpha_{d}}$. The exponent $\alpha_{d}$ is a parameter that quantifies the long-range power-law correlation properties of the signal. Values of $\alpha_{\mathrm{d}}<$ 0.5 indicate the presence of anti-correlations in the time series, $\alpha_{\mathrm{d}}=0.5$ absence of correlations (white noise) and $\alpha_{\mathrm{d}}>0.5$ indicates the presence of positive correlations in the time series.

Conditional probabilities analysis. The conditional probability of an event $H$ for a given event $X$ is defined as follows:

$$
P(H \mid X)=\frac{P(H \cap X)}{P(X)},
$$

where $P(H \cap X)$ is the probability that $H$ and $X$ jointly occur, and $P(X)>0$ is the probability of the event $X$. The condition $X$ reduces the statistics and increases the fluctuations of the distribution $P(H \mid X)$ compared with $P(H)$. The following error is associated to each bin of the densities (Corral, 2006): $\epsilon_{H}=\frac{1}{d H} \sqrt{\frac{p(1-p)}{N}}$, where $p=P(H) d H$ is the probability to observe a $H$ in the range $H, H+d H$ and $N$ is the total number of events. From probability theory $P(H \mid X)=P(X)$ if and only if $H$ does not depend on $X$. On the contrary, $P(H \mid X) \neq P(X)$ implies that $H$ and $X$ are not independent of each other, and their relation can be quantified by a suitable correlation measure. In the analysis of burst coupling (see Results, Anti-correlated coupling between the durations of consecutive $\theta$ and $\delta$-bursts), $H$ and $X$ are considered significantly correlated if $P(H \mid X)-P(X)>\epsilon_{H}$.

Software accessibility. Software used for data preprocessing is available upon request.

\section{Model of anti-correlated burst coupling}

The model consists of the following steps.

Random drawing and ranking. $N$ durations $d_{\theta}$ and $d_{\delta}$ are randomly drawn from the empirical distributions previously obtained using a specific window size $w . d_{\theta}$ and $d_{\delta}$ are separately sorted in ascending order, i.e., from shortest to longest, and get a distinct ordinal numbers from $k=$ $1,2, \ldots, N$, which corresponds to their rank. This procedure ensures that each duration has a unique rank. The ranked $d_{\theta}$ and $d_{\delta}$ are then paired with a tunable degree of anti-correlation and a new time series of alternating $\theta$ - and $\delta$-burst durations is thus generated. The coarse-grained properties of the resulting time series depends on the degree of anticorrelations used in the pairing.

Correlated pairing. Once $d_{\theta}$ and $d_{\delta}$ are ranked and a distinct, unique ordinal number is associated to them, one randomly choose a $d_{\theta}$ with rank $k_{1}$ between 1 and $N$. To choose the following $d_{\delta}$, one draws a random number $k_{2}$ from a Gaussian distribution with mean $\mu=1+N-k_{1}$ and $\operatorname{SD} \sigma$, and takes $d_{\delta}$ as the duration corresponding to rank $k_{2}$. This procedure is iterated $N$ times, and at each iteration $i$ the mean of the Gaussian from which one draws the next random rank, $k_{i}$, depends on $k_{i-1}$, i.e., $\mu-1+N-k_{i-1}$. At each iteration, $k_{i}$ will correspond to a duration $d_{\delta}$ from the sorted $\delta$-burst durations if the preceding burst was a $\theta$-burst with duration $d_{\theta}$, whereas $k_{i}$ will select a duration $d_{\theta}$ from the sorted $\theta$-burst durations if the preceding burst was a $\delta$-burst with duration $d_{\delta}$. As a result one obtains a sequence of $d_{\theta}$ and $d_{\delta}$ whose degree of anticorrelations is controlled by a single parameter, $\sigma$. The smaller $\sigma$, the stronger anti-correlations are.

Binary series and coarse-graining. To characterize the coarse-grained properties, the time series is first converted in a binary sequence, namely a sequence of "+" and "-". Because each duration is by definition a multiple $n$ of the unit window $w$, namely $d=n w$, the $n$ windows belonging to a $d_{\theta}$ are populated with + , whereas the $n$ windows belonging to a $d_{\delta}$ with - (see Fig. 14c). As a result one has a sequence of windows populated with + and - . This binary sequence is then coarse-grained grouping a given number $\Delta$ of consecutive windows, with $\Delta$ odd number, and assigning + or - to the new windows of size $\Delta$ according to a majority rule, i.e., one assign $+(-)$ if the number of + is larger (smaller) than the number of - (see Fig. 14). A coarse-grained binary sequence (CGBS) is thus obtained, and $d^{C G}$ coarse-grained durations are calculated as shown in Figure 14.

\section{Results}

Transient dynamics in bursting activity of $\boldsymbol{\theta}$ and $\delta$ rhythms

Statistics of sleep and wake bout durations show that control rats spend on average $50.47 \pm 3.37 \%$ of the time in wakefulness, $42.12 \pm 2.39 \%$ in NREM sleep, and $7.41 \pm 1.09 \%$ in REM sleep in the $24 \mathrm{~h}$ period. Correspondingly, rats with VLPO lesion spend on average more time in wakefulness $(61.46 \pm 4.17 \%)$, and experience a corresponding decrease in the percentage of NREM $(31.94 \pm 3.85 \%)$ and REM sleep $(6.60 \pm 0.86 \%)$ across the $24 \mathrm{~h}$ period, in agreement with previous reports ( $\mathrm{Lu}$ et al., 2000; Vetrivelan et al., 2012). The analysis of power spectra (Fig. 1c) for control rats indicates dominant delta rhythm in NREM and increasing spectral power in the theta band for REM and WAKE, and a decrease in relative delta power during NREM in VLPOlesioned rats (Lu et al., 2000; Vetrivelan et al., 2012). However, total sleep-wake time, average bout duration, and spectral power reflect static characteristics of the sleep process. In contrast, to investigate emergent signatures of criticality in sleep microarchitecture, we focus on dynamical characteristics of dominant cortical rhythms at short time scales.

To dissect the temporal organization of $\delta$ and $\theta$-bursts in the broadband brain activity across the sleep-wake cycle, we analyze the time evolution of the EEG signal by evaluating the spectral power in several frequency bands on non-overlapping windows of length $w$ (Fig. 1a,b; see Materials and Methods, Data analysis). Figure $1 d$ shows a typical spectrogram $S(f)$ as a function of time for a $2 \mathrm{~h}$ lights-on recording of a rat in the control group. In each window, the spectral power is primarily concentrated in either the $\delta$-wave $(0-4 \mathrm{~Hz})$ or the $\theta$-wave $(4-8 \mathrm{~Hz})$ band, and exhibits sharp transitions from periods with dominant $\delta$ to periods with dominant $\theta$ waves. In particular, during wake periods most of the windows in the spectrogram exhibit dominant $\theta$ band (Fig. 1e), with fewer transitions to periods with dominant $\delta$ waves. Such dynamics can be understood as the temporal evolution of the ratio $R_{\theta \delta}=S(\theta) / S(\delta)$ between $\theta$ and $\delta$ spectral power in association with different physiological states; NREM, REM, and arousals/wake (Fig. 1 shows the transient dynamics of $\delta$ - and $\theta$-wave power represented by the logarithm of $R_{\theta \delta}$ as a function of time $t$ ).

The ratio $R_{\theta \delta}(t)$ exhibits irregular, intermittent fluctuations between values larger and smaller than a threshold $T h$, a typical characteristic of non-equilibrium dynamics: $R_{\theta \delta}>T h=1$ indicates that the spectral power in the $\theta$-wave band is dominant; vice versa, for $R_{\theta \delta}<T h=1$ the spectral power is dominated by the $\delta$-wave (Fig. $1 d, e$ ). We define bursts in $\theta$ and $\delta$ rhythms as sequences of consecutive time windows where $R_{\theta \delta}>T h=1$ and $R_{\theta \delta}<T h=1$, respectively (Fig. $1 f$ ). We associate a duration $d=n \times w$ to each burst (see Materials and Methods, Data analysis), where $n$ is the number of consecutive windows belonging to a given burst and $w$ is the window length (Fig. $1 f$ ).

\section{Distinct functional forms of $\boldsymbol{\theta}$ - and $\boldsymbol{\delta}$-burst duration distributions indicative of self-organization at criticality} We next study the probability distribution of the durations of $\theta$ and $\delta$-bursts over a $24 \mathrm{~h}$ period for control and VLPO-lesioned rats (see Materials and Methods, Experimental setup). We notice that $\theta$ - and $\delta$-bursts follow very different statistics. The distribution $P_{\theta}$ of $\theta$-burst durations exhibits power-law behavior followed by a cutoff (Fig. $2 a$ ), $P_{\theta}(d) \propto d^{-\alpha}$, where $\alpha$ denotes the scaling exponent of the power-law. Power law distributions $P(x) \propto x^{-\alpha}$ are the statistical hallmark of scale invariance, i.e., they are not altered by a change of scale from $x$ to $L x$, and depending on the context, they imply that events of any size, length, or 

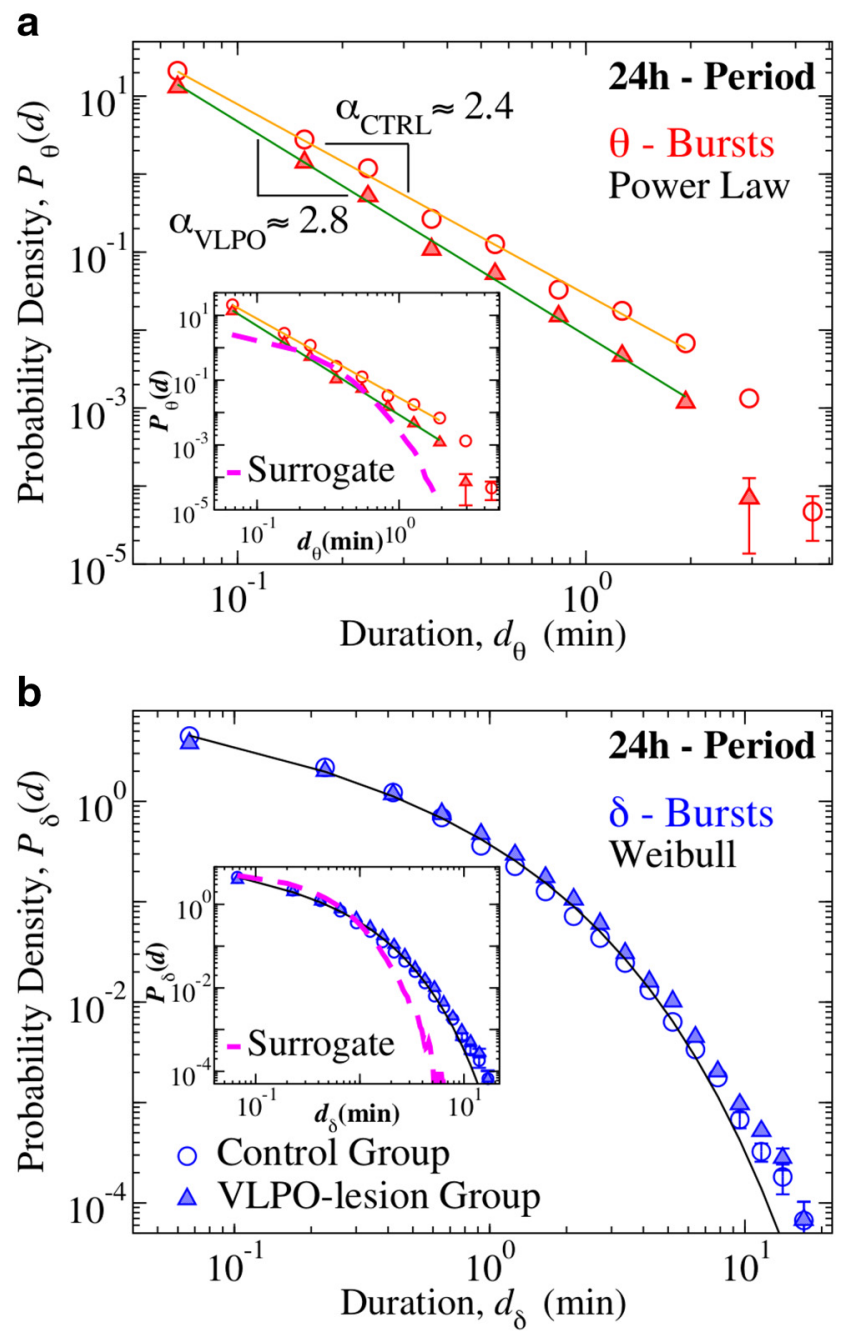

Figure 2. Durations of $\theta$ and $\delta$-bursts across the $24 \mathrm{~h}$ sleep-wake cycle follow different statistics that are modulated by VLPO. $\boldsymbol{a}$, Distribution of $\theta$-burst durations for control (open circles) and VLPO lesioned rats (full triangles) over the $24 \mathrm{~h}$ period (pooled data, 6 control and 6 VLPO-lesioned rats). The distribution exhibits a power-law behavior in both groups (colored tick lines). Lesions in the VLPO area cause a significant increase in the power-law exponent from $\alpha_{\text {CTRL }}=2.44 \pm 0.07$ to $\alpha_{\text {VLP0 }}=2.75 \pm 0.05$ (exponent \pm error on fit), indicating a reduced probability for long $\theta$-bursts (Group comparison, $t$ test, $p=0.0005$ ). $\boldsymbol{b}$, Distribution of $\delta$ burst durations for control (open circles) and VLPO lesioned rats (full triangles) over $24 \mathrm{~h}$ period (pooled data). In contrast to the statistics of $\theta$-bursts, $\delta$-bursts durations follow Weibull distributions. Weibull parameters are not significantly affected by lesions of the VLPO ( $\beta_{\text {CTRL }}=0.64$, $\lambda_{\text {CTRL }}=0.38 ; \beta_{\text {VLPO }}=0.70, \lambda_{\text {VLPO }}=0.54$ ). The black tick line is a Weibull fit of the distribution for control rats. All durations are calculated using a window size $w=4 \mathrm{~s}$ and threshold Th $=1$ on the ratio $R_{\theta \delta}$ (Fig. 1). Insets, Distributions of surrogate $\theta$ - and $\delta$-burst durations markedly deviate from the original distributions. Error bars $\delta P$ are calculated for each value of the distributions as $\delta P=(\sqrt{p(1-p) / N}) / d D$, and where not shown are smaller than the symbol size. Error bar calculation and binning procedure are described in Materials and Methods, Data analysis.

duration are likely to occur with some finite probability that is larger than expected in a random or short-range correlated process. Presence of power-law indicates absence of characteristic time scales in the underlying dynamics, which is a typical feature of physical systems at the critical point of continuous phase transition; a highly sensitive state where cooperative behavior spontaneously emerges over a range of time scales characterized by long-range correlations. Importantly, the scale-invariant powerlaw behavior characterizing the distribution of $\theta$-burst durations is significantly influenced by lesions in the VLPO. Indeed, we find that $\alpha \simeq 2.4$ in control rats, whereas $\alpha \simeq 2.8$ in VLPO-lesioned rats (Fig. 2a). The power-law is consistent across rats in both groups, and the average exponent in the VLPO-lesioned group is significantly higher than the exponent in the control group $\left[\alpha_{\mathrm{CTRL}}=2.29 \pm 0.15\right.$ and $\alpha_{V L P O}=2.71 \pm 0.11($ mean $\pm \mathrm{SD}) ; t$ test, $p=0.0005]$. An increase in the power-law exponent indicates that bilateral lesions of the VLPO alter the dynamical microarchitecture of $\theta$-bursts across the $24 \mathrm{~h}$ sleep-wake cycle, leading to a decreased likelihood of long lasting $\theta$-bursts; an effect not previously observed.

In contrast to the power-law feature of $\theta$-burst, the statistics of $\delta$-burst duration follows a different behavior that is described by a Weibull distribution $P_{\delta}(d ; \lambda, \beta)=\frac{\beta}{\lambda}\left(\frac{d}{\lambda}\right)^{\beta-1} e^{-(d / \lambda)^{\beta}}$, where $\lambda$ indicates the characteristic time scale, and $\beta$ is the shape parameter (Fig. 2b). Further, we find that the distribution of $\delta$-burst durations follows the same Weibull functional form for both control and VLPO-lesioned groups, with similar values of the parameters $\lambda$ and $\beta$.

The functional forms established for the distributions of $\theta$ and $\delta$-burst durations in Figure 2, indicate a very different temporal organization of $\theta$ - and $\delta$-bursts. A surrogate test based on randomizing the sequence of windows $w$ in the EEG spectrogram (Fig. 1d) leads to exponentially distributed $\theta$ - and $\delta$-burst (Fig. 2, insets), and shows that the observed temporal organization in bursting activity of brain rhythms is physiologically relevant and relates to underlying regulation.

The results demonstrate a remarkable duality of scale-free, power-law dynamics for $\theta$-bursts, and Weibull dynamics with characteristic time scale for $\delta$-bursts. The coexistence of scalefree $\theta$-bursts and Weibull distributed $\delta$-bursts appears to be a general feature of cortical activity across the entire sleep-wake cycle of individual subjects in each group, and it is preserved after major lesions of the VLPO area. Remarkably, VLPO lesions, although significantly affecting sleep statistics (Lu et al., 2000; Vetrivelan et al., 2012), do not disrupt the fundamental duality of power-law and Weibull. Coexistence of scale-invariant and exponential-like behaviors is a hallmark of self-organization at criticality in non-equilibrium systems characterized by alternating active and inactive states; unlike systems at equilibrium, such systems maintain critical behavior without external tuning (Boffetta et al., 1999; Paczuski et al., 2005; Munoz, 2018). Thus, our observations point to an intrinsic common mechanism that underlies the temporal organization of bursting activity in both $\delta$ and $\theta$ cortical waves across the distinct physiological states of wake/arousals and sleep. Importantly, the deviation of the VLPO-lesioned $\theta$-burst power-law exponent from the value measured in control rats, indicates that lesion of VLPO neurons alters the optimal underlying dynamics for sleep regulation.

To better describe bursting activity of $\theta$ - and $\delta$-waves across the sleep-wake cycle, and the role of the VLPO neuronal population in this dynamics, we next analyze the distributions of $\theta$ and $\delta$-burst durations separately during the $12 \mathrm{~h}$ dark/light periods. Although sleep and wake are characterized by different dominant brain rhythms with distinct dynamics, synchronization and coupling patterns across cortical areas (Kopell et al., 2000; Liu et al., 2015), our analysis shows that the duality of power-law and Weibull for $\theta$ - and $\delta$-bursts duration distributions is robust and independent of the dominant physiologic state. Indeed, such coexistence appears to be a basic characteristic of cortical activity during both dark and light periods (Fig. 3). Importantly, the scaling exponent $\alpha$ of the power-law as well as the Weibull pa- 

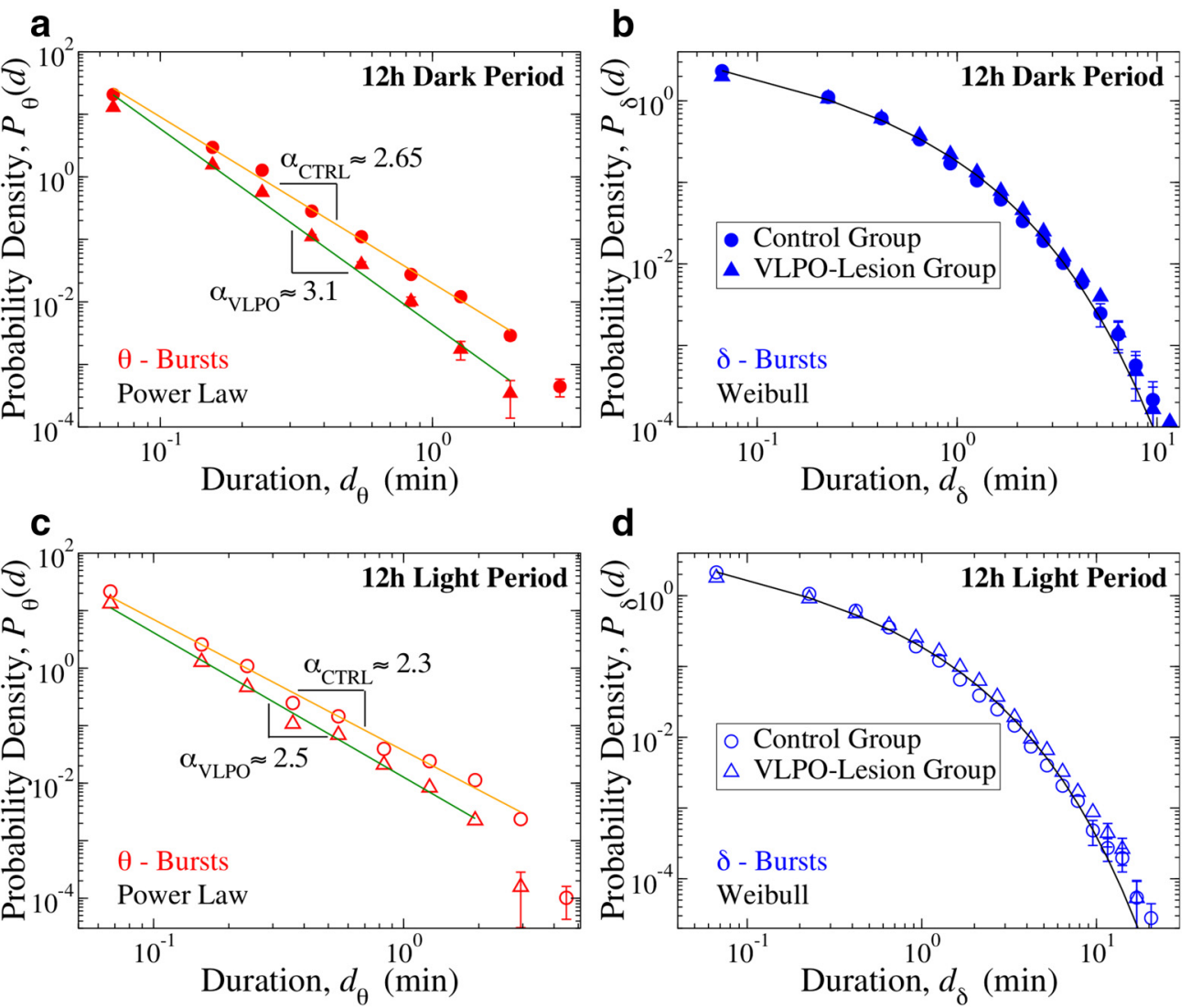

Figure 3. Critical behavior represented by duality of power-law and Weibull distribution for $\theta$ - and $\delta$-bursts characterizes cortical activity during both dark and light periods. $\boldsymbol{a}$, Probability distributions of $\theta$-burst durations for control (full circles) and VLPO-lesioned (full triangles) rats over the $12 \mathrm{~h}$ dark (lights-off) period (pooled data, 6 control and 6 VLP0-lesioned rats) follow a power-law with exponent $\alpha_{\text {CTRL }}=2.66 \pm 0.07$ and $\alpha_{\text {VLPO }}=3.13 \pm 0.12$ (exponent \pm error on fit). Both groups exhibit an exponent larger than in the $24 \mathrm{~h}$ sleep-wake cycle (Fig. 2), in particular VLPO-lesioned rats, and the difference between the two groups becomes more pronounced (Control vs VLP0 group comparison: $t$ test, $p=0.003$ ). $\boldsymbol{b}$, Probability distributions of $\delta$-burst durations for control (full circles) and VLPO-lesioned (full triangles) rats over $12 \mathrm{~h}$ dark (lights-off) period (pooled data) follow a Weibull behavior in both groups, with no significant differences in the fitting parameters $\left(\beta_{\text {CTRL }}=0.66, \lambda_{\text {CTRL }}=0.36 ; \beta_{\text {VLPO }}=0.72, \lambda_{\text {VLPO }}=0.49\right.$ ). c, Probability distributions of $\theta$-burst durations for control (open circles) and VLP0-lesioned (open triangles) rats over the $12 \mathrm{~h}$ lights-on period (pooled data, 6 control and 6 VLPO-lesioned rats) also follow a power-law, but exponents are smaller than in the dark period, with $\alpha_{\text {CTRL }}=2.28 \pm 0.09$ and $\alpha_{\text {VIPO }}=2.51 \pm$ 0.07 . The difference between the two groups is less pronounced than in dark period (Control vs VLPO comparison: $t$ test, $p=0.131$ ). Paired tests show significant differences between dark and light periods in each group: control dark versus control light $t$ test, $p<0.0005$; VLPO dark vs VLPO light $t$ test, $p=0.0010$. $\boldsymbol{d}$, Probability distributions of $\delta$ burst durations for control (open circles) and VLPO lesioned (open triangles) rats over $12 \mathrm{~h}$ lights-on period (pooled data) also follow a Weibull behavior, with no significant differences in the fitting parameters $\left(\beta_{\text {CTRL }}=0.70, \lambda_{\text {CTRL }}=0.37\right.$; $\beta_{\text {VLP0 }}=0.67, \lambda_{\text {VLPO }}=0.62$ ). Black tick lines in c and $\boldsymbol{d}$ show Weibull fits for the distribution of the control group. All durations are calculated using a window size $w=4 \mathrm{~s}$ and threshold Th $=1$ on the ratio $R_{\theta \delta}$ (Fig. 1). Error bars are calculated for each value and where not shown are smaller than the symbol size. Error bars $\delta P$ are calculated for each value of the distributions as $\delta P=(\sqrt{p(1-p) / N}) / d D$, and where not shown are smaller than the symbol size. Error bars calculation and binning procedure are described in Materials and Methods, Data analysis.

rameters $\lambda$ and $\beta$ concurrently change comparing dark to light periods, indicating a coordinated modulation of $\theta$ and $\delta$ wave dynamics across sleep and wake. In contrast to humans, rats are predominantly awake during the dark period and asleep during the light period. We observe that $\alpha$ is larger in the dark than in the light period (Fig. $3 a, c)$, indicating that long $\theta$-bursts are more likely when rats are predominantly asleep. The higher probability of longer-lasting $\theta$-bursts during the light period could be associated with the presence of longer episodes of REM sleep, when $\theta$-waves are dominant. This leads to an average increase of the power in the $\theta$ band and thus to a higher likelihood for longer $\theta$-bursts during the light periods. For control rats, we find that the power-law exponent decreases from $\alpha \approx 2.65$ during dark to $\alpha \approx 2.5$ during light (Fig. $3 a$ ). This variation is more pronounced in VLPO-lesioned rats, where the exponent decreases from $\alpha \approx$ 3.1 during dark to $\alpha \approx 2.5$ during light periods (Fig. 3c), and may be related to the average reduction of REM sleep. These results are consistent across rats both during dark $\left[\alpha_{\text {CTRL }}=2.62 \pm 0.18\right.$; $\alpha_{\mathrm{VLPO}}=3.10 \pm 0.24$ (mean $\pm \mathrm{SD}$ ); $t$ test, $p=0.003$ ] and light $\left[\alpha_{\mathrm{CTRL}}=2.14 \pm 0.13 ; \alpha_{\mathrm{VLPO}}=2.29 \pm 0.15\right.$ (mean $\left.\pm \mathrm{SD}\right) ; t$ test, $p=0.131]$, and show significant difference particularly for the $12 \mathrm{~h}$ dark period. Paired tests also show significant differences between dark and light periods in each group (Control dark vs control light $t$ test, $p=0.0005$; VLPO dark vs VLPO light $t$ test, $p=0.0010)$. The comparison between control and VLPOlesioned rats shows that VLPO lesions affect the distributions of $\theta$-burst durations, in particular during the dark period, while having no particular influence on the distributions of $\delta$-burst durations, which overlap within error bars (Fig. 3). This indicates that, although lesions of VLPO neurons reduce $\delta$ power and total sleep time (Lu et al., 2000; Vetrivelan et al., 2012), they do not alter the dynamics of $\delta$-bursts, but instead influence the powerlaw organization in $\theta$-burst dynamics and lead to an increase of the power-law exponent, indicating a decreased likelihood for long-lasting bursts and a higher probability to observe shorter bursts; evidence of fragmentation of bursting activity in the $\theta$-wave band that parallels the increased fragmentation of sleep in VLPO-lesioned rats.

Previous studies have shown that preceding sleep-wake history affects sleep consolidation and slow-wave activity. In par- 

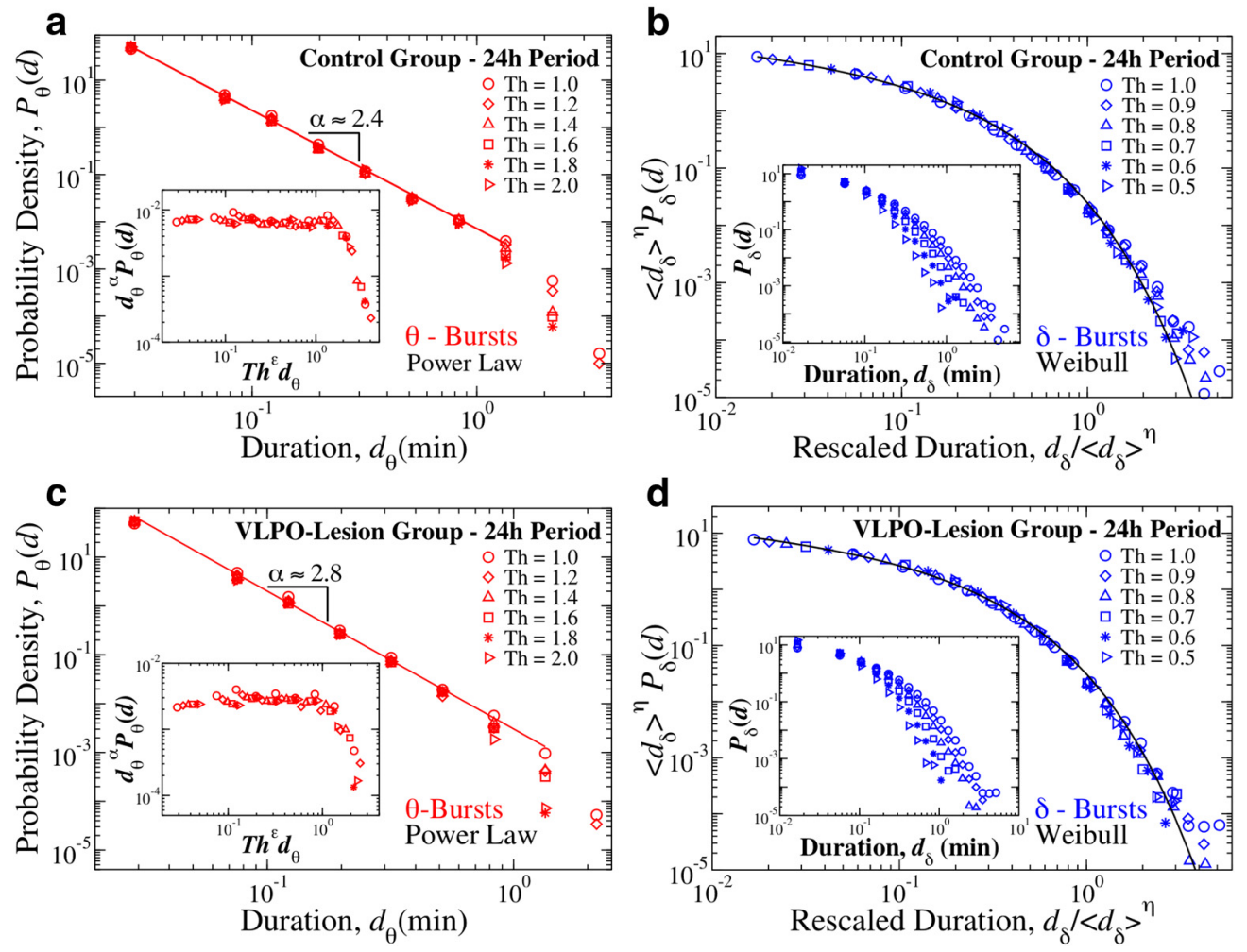

Figure 4. Critical characteristics in temporal dynamics of bursts in dominant rhythms are a fundamental feature of cortical activity across the sleep-wake cycle, independent of thresholds used to define bursts. The functional form of $\theta$ - and $\delta$-burst duration distributions is preserved for different threshold values Th imposed on the ratio $R_{\theta \delta}$ (Fig. 1). $\boldsymbol{a}$, Probability distributions of $\theta$-burst durations for control rats over a $24 \mathrm{~h}$ period (pooled data) evaluated using different $T h$ values consistently follow the same power law behavior (red line), with a cutoff that is controlled by $T h$. With increasing $T h$ the distribution cutoff shifts toward shorter burst durations. Inset, Data for different $T h$ collapse onto a single universal function $f_{\theta}$ when we plot $P(d) d^{\alpha}$ versus $T h^{\epsilon} d$, with $\alpha=2.4$ and $\boldsymbol{\epsilon}=0.8 . \boldsymbol{b}$, Rescaled distribution of $\delta$-burst durations for control rats over a $24 \mathrm{~h}$ period (pooled data) obtained for different $T$ h values collapse onto a single function following a Weibull behavior $f(d ; \lambda, \beta)$ (black line), with $\beta=0.71$ and $\lambda=0.11$. Distributions are rescaled by $\left\langle d_{\delta}\right\rangle^{\eta}$, where $\left\langle d_{\delta}\right\rangle$ is the mean $\delta$-burst duration and $\eta=1.3$. Inset, Distributions $P_{\delta}$ for different thresholds (not rescaled).c, Probability distributions of $\theta$ burst durations for VLPO-lesioned rats over a $24 \mathrm{~h}$ period (pooled data) evaluated using different $T$ Th values follow the same power law behavior, with a cutoff controlled by Th. Inset, Data collapse onto a single function $f_{\theta}$ by plotting $P(d) d_{\alpha}$ versus $T h^{\epsilon} d$ with $\alpha=2.8$ and $\boldsymbol{\epsilon}=0.8$ (same as for control rats in $\boldsymbol{a}$ ). $\boldsymbol{d}$, Rescaled distribution of $\delta$-burst durations for VLPO-lesioned rats over a $24 \mathrm{~h}$ period (pooled data) obtained for different Th values collapse onto a single Weibull distribution $f(d ; \lambda, \beta)$ (black line) with $\lambda=0.12$ and $\beta=0.72$. Distributions are rescaled by. $\left\langle d_{\delta}\right\rangle^{\eta}$., with $\eta=1.3$. Inset, Distributions $P_{\delta}$ for different thresholds $T h$ (not rescaled). Results in all panels are obtained for a fixed scale of analysis, keeping the window size $w=4 \mathrm{~s}$ (Fig. 1). Results are consistent when considering separately light and dark periods (Figs. 5, 6).

ticular, sleep deprivation increases sleep consolidation and continuity, reduces frequency of short wake episodes, and thus sleep fragmentation (Trachsel et al., 1991). Moreover, it has been shown that after long periods of sleep deprivation NREM episodes tend to be longer, with a larger amount of slow-wave sleep and increased EEG amplitude (Tobler and Borbély, 1986; Franken et al., 1991; Rodriguez et al., 2016). In this context, it would be important to understand how change in homeostatic drive (for example in response to sleep deprivation) would impact the reported underlying critical dynamics of cortical rhythms. While the established non-equilibrium dynamics in cortical rhythms at small time scales apparently contrast homeostatic regulation of the sleep-wake cycle (Borbély and Achermann, 1999; Achermann and Borbély, 2003), the interplay between this two mechanisms remains to be better investigated and understood in future studies. VLPO-lesioned rats, although exhibiting an increase in the percentage of wake across the $24 \mathrm{~h}$ period and in average duration of wake bouts (Lu et al., 2000; Vetrivelan et al., 2012), do not show extended periods of wake comparable to those used in previous experimental protocol for studying sleep deprivation (Tobler and Borbély, 1986; Franken et al., 1991; Trachsel et al., 1991), and therefore we cannot not draw any conclusion on the effect of homeostatic mechanisms on the underlying critical dynamics based on the data analyzed here.
Furthermore, loss of VLPO neurons also disinhibit the wakepromoting system including hypocretin system, and leads to their increased activity (Lu et al., 2000; Vetrivelan et al., 2012). These changes are interrelated, and may be difficult to dissociate from each other. For example, changes in EEG spectra (Fig. 1) and sleep fragmentation after VLPO lesions (Lu et al., 2000; Vetrivelan et al., 2012) could also be due to increased activity of hypocretin system (Sakurai, 2007). On the other hand, other manipulations, e.g., water/food availability, LD conditions, ambient temperature etc., could also alter EEG and sleep-wake cycle by altering VLPO neuron activity, and therefore influence the $\theta$ - and $\delta$-burst dynamics. Our current study investigated the intrinsic properties of cortical dynamics following specific VLPO lesions, and future investigations are necessary to understand the mechanisms (changes in VLPO neural activity) by which other manipulations alter cortical dynamics, and in particular affect the ability of the system to self-tune at criticality.

\section{Robust scale-invariant critical behavior of $\boldsymbol{\theta}$ - and $\boldsymbol{\delta}$-bursts across time scales}

We have shown that bursts associated with $\theta$ and $\delta$ rhythms exhibit a distinct temporal organization that is captured by specific duration distributions: a power-law for $\theta$-bursts, indicating 



Figure 5. Distribution of $\theta$ - and $\delta$-burst durations in the dark period are independent of the specific threshold Th used to identify bursts and can be described by unique scaling functions. Distribution of $\theta$-and $\delta$-burst durations in the dark period for different threshold values $T$ h on the ratio $R_{\theta \delta}$ and window size $w=4 \mathrm{~s}$. $\boldsymbol{a}$, Distribution of $\theta$ burst durations for control rats over a $12 \mathrm{~h}$ dark period (pooled data). Distributions evaluated using different Th values consistently follow the same power law behavior, with a cutoff that is controlled by Th. Inset, The data collapse onto a universal function $f_{\theta}$ when we plot $P(d) d^{\alpha}$ versus $T h^{\epsilon} d$, with $\alpha=2.65$ and $\boldsymbol{\epsilon}=0.8$. $\boldsymbol{b}$, Rescaled distribution of $\delta$-burst durations for control rats over a $12 \mathrm{~h}$ dark period (pooled data). Distributions are rescaled by $\left\langle d_{\delta}\right\rangle^{\eta}$, with $\left\langle d_{\delta}\right\rangle$ mean $\delta$-burst duration and $\eta=1.3$. After rescaling, distributions collapse onto a unique function $f_{\delta}$ that is well described by a Weibull distribution $f(d ; \lambda, \beta)$ (thick line), with $\lambda=0.01$ and $\beta=0.72$. Inset, Distributions $P_{\delta}$ for different thresholds (not rescaled). c, Distribution of $\theta$ burst durations for VLP0-lesioned rats over a 12 h period (pooled data). Distributions evaluated using different $T h$ values consistently follow the same power law behavior, with a cutoff that is controlled by $T$. Data collapse onto a universal function $f_{\theta}$ by plotting $P(d) d^{\alpha}$ versus $T h^{\epsilon} d$ with $\alpha=3.1$ and $\epsilon=0.8$. $d$, Rescaled distribution of $\delta$ burst durations for VLP0-lesioned rats over a $12 \mathrm{~h}$ dark period (pooled). Distributions are rescaled by $\left\langle d_{\delta}\right\rangle \eta^{\eta}$, with $\eta=1.3$. After rescaling, the distributions collapse onto a unique function $f_{\delta}$ that is close to a Weibull distribution $f(x ; \lambda, \beta)$ (thick black line), with $\lambda=0.05$ and $\beta=0.75$. Inset, Distributions $P_{\delta}$ for different thresholds (not rescaled).

absence of a characteristic time scale, and a Weibull for $\delta$-bursts, with a characteristic time scale $\lambda$ (Figs. 2, 3). These findings are based on a particular observational window size $w$ and threshold Th that were used to analyze bursting activity (Fig. 1; see Materials and Methods, Data analysis). To demonstrate that our results are independent of the particular choice of $T h$ and $w$, we repeat the analyses for a range of parameter values. We find that the dynamics of burst durations across the $24 \mathrm{~h}$ sleep-wake cycle is indeed described by unique scaling functions.

We first examine the duration distributions of $\theta$ - and $\delta$-bursts for different threshold values Th, keeping the window size $w$ fixed. By increasing the threshold on the ratio $R_{\theta \delta}$ from $T h=1$ to $T h=2$, we find that the scaling exponent $\alpha$ characterizing the power-law distribution of $\theta$-burst durations remains stable (data collapse onto a single curve; Fig. $4 a, c$ ). The scaling behavior is followed by a cutoff that, with increasing Th values, shifts to shorter burst durations $d_{\theta}$. For both control and VLPO-lesioned rats this behavior can be expressed in terms of the following scaling relation:

$$
P_{\theta}(d)=d^{-\alpha} f_{\theta}\left(d / T h^{-\epsilon}\right) .
$$

Where $\alpha$ is the power-law scaling exponent, $f\left(d / T h^{-\epsilon}\right)$ is a scaling function, and $\epsilon$ expresses the dependence of the cutoff on $T h$. The existence of a scaling function $f\left(d / T h^{-\epsilon}\right)$ satisfying Equation 6 is confirmed by the data collapse obtained by plotting $P(d) d^{\alpha}$ versus $T h^{\epsilon} d$ for several values of $T h$ (Fig. $4 a, c$, insets).
Similarly, we show that the distribution of $\delta$-burst durations is independent of the threshold $T h$, and is described by a single scaling function (Fig. $4 b, d$ ). Because a $\delta$-burst is defined as a time period of consecutive windows $w$ where $R_{\theta \delta}<T h=1$ (Fig. $1 f)$, to properly explore the behavior of the duration distribution for states with increasingly dominant $\delta$ power, we repeat the analysis for different values $T h<1$. We observe that, as $T h$ decreases, the probability for long $\delta$-bursts decreases, while short $\delta$-bursts become more likely (Fig. $4 b, d$, insets). However, when distributions are rescaled by their respective mean $\delta$-burst duration $\left\langle d_{\delta}\right\rangle$, they all collapse onto a unique function $f_{\delta}$, which is the same for both control and VLPO-lesioned rats (Fig. $4 b, d$, respectively). Such function is defined by the following scaling relation:

$$
P_{\delta}(d)=\left\langle d_{\delta}\right\rangle^{-\eta} \cdot f_{\delta}\left(d /\left\langle d_{\delta}\right\rangle^{\eta}\right)
$$

where $\eta=1.3$ for both rat groups, and is well described by a Weibull functional form, as found by rescaling $d_{\delta}$ and $P_{\delta}$ (Fig. $4 b, d$ ).

Repeating the analysis for $12 \mathrm{~h}$ dark/light periods separately, we find that Equations 6 and 7 consistently describe the dynamics of $\delta$ - and $\theta$-bursts in both control and VLPO-lesioned groups (Figs. 5, 6).

Thus, our results indicate that the duality of power-law and Weibull behavior, as well as the scaling properties summarized in Equations 6 and 7, are robust features of the bursting activity 

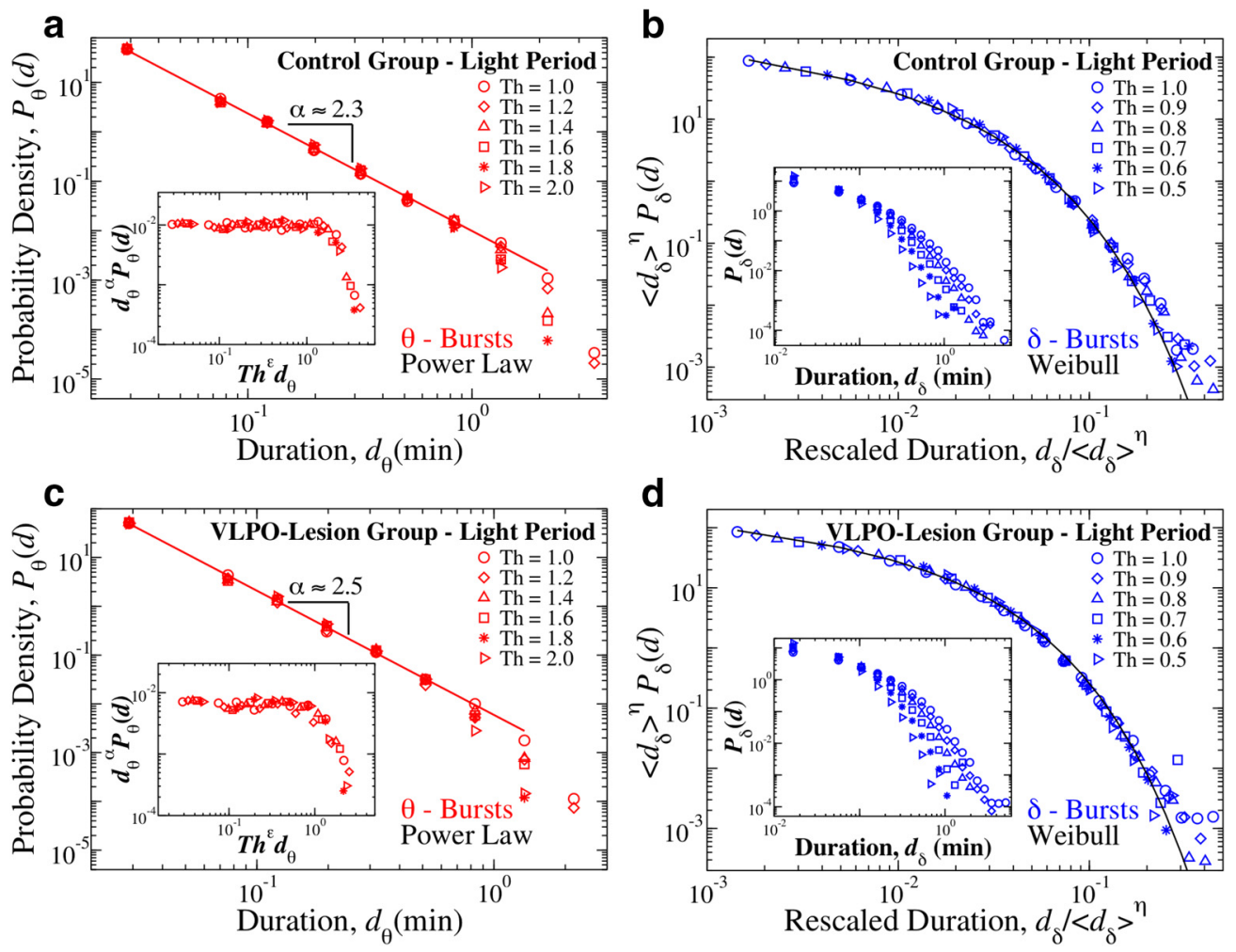

Figure 6. Distribution of $\theta$ - and $\delta$-burst durations in the light period are independent of the specific threshold Th used to identify bursts and can be described by scaling functions. Distribution of $\theta$ and $\delta$ burst durations in the light period for different threshold values Th on the ratio $R_{\theta \delta}$ and window size $w=4$ s. $a$, Distribution of $\theta$ burst durations for control rats over a $12 \mathrm{~h}$ light period (pooled data). Distributions evaluated using different $T h$ values consistently follow the same power law behavior, with a cutoff that is controlled by $T h$. The data collapse onto a universal function $f_{\theta}$ when we plot $P(d) d^{\alpha}$ versus $T h^{\epsilon} d$, with $\alpha=2.3$ and $\epsilon=0.8$ (inset). $\boldsymbol{b}$, Rescaled distribution of $\delta$ burst durations for control rats over a $12 \mathrm{~h} \mathrm{light} \mathrm{period} \mathrm{(pooled} \mathrm{data).} \mathrm{Distributions} \mathrm{are} \mathrm{rescaled}$ by $\left\langle d_{\delta}\right\rangle^{\eta}$, with $\left\langle d_{\delta}\right\rangle$ mean $\delta$-burst duration and $\eta=1$.3. After rescaling, distributions collapse onto a unique function $f_{\delta}$ that is close to a Weibull distribution $f(d ; \lambda, \beta)$ (black line), with $\lambda=$ 0.04 and $\beta=0.70$. Inset, Distributions $P_{\delta}$ for different thresholds (not rescaled). $c$, Distribution of $\theta$ burst durations for VLPO-lesion rats over a $12 \mathrm{~h}$ period (pooled data). Distributions evaluated using different $T h$ values consistently follow the same power law behavior, with a cutoff that is controlled by $T$. Inset, Data collapse onto a universal function $f_{\theta}$ by plotting $P(d) d^{\alpha}$ versus $T h^{\epsilon} d$ with $\alpha=2.5$ and $\epsilon=0.8$. $\boldsymbol{d}$, Rescaled distribution of $\delta$ burst durations for VLPO-lesion rats over a $12 \mathrm{~h}$ light period (pooled). Distributions are rescaled by $\left\langle d_{\delta}\right\rangle^{\eta}$, with $\eta=1.3$, and collapse onto a unique function $f_{\delta}$ that is close to a Weibull distribution $f(d ; \lambda, \beta)$ (thick black line), with $\lambda=0.05$ and $\beta=0.74$. Inset, Distributions $P_{\delta}$ for different thresholds (not rescaled).

across the sleep-wake cycle, and do not depend on the particular threshold Th used to study the dynamics of $\theta$ - and $\delta$-bursts.

Furthermore, we find that the functional behavior of the distribution of $\theta$ - and $\delta$-burst durations is to a large extent also independent of the window size $w$, used to investigate the time course of the EEG spectral power. Intuitively, a larger $w$ would tend to fail in identifying short bursts and merge them together, thus causing an increase in the probability of observing longer durations. In that regard, considering the power-law temporal organization of $\theta$-bursts, larger window sizes $w$ mainly influence the tail of the distribution with longer durations, thus leading to a decrease of the scaling exponent $\alpha$ (Fig. 7a,c, insets). We note that the window size effect becomes visible only for extremely large $w$ compared with the average $\theta$-burst duration $\left\langle d_{\theta}\right\rangle$. However, when the $\theta$-burst durations are rescaled by the window size $w$, all distributions collapse onto a single power-law (Fig. 7a,c), confirming the robustness of the results obtained in Figure $2 a$ for both control and VLPO-lesioned rats. Such rescaling is defined by the following relation:

$$
P_{\theta}(d) \sim w^{-1} \cdot f_{\theta}(d / w) .
$$

Separate analyses of $12 \mathrm{~h}$ dark/light periods for different window sizes $w$ (Figs. $8 a, c$, Fig. $9 a, c$ ) further confirm the robustness of the established power-law behavior for the $\theta$-burst durations (Fig. 3a,c).
A similar data collapse characterizes the dependence of the $\delta$-burst duration distribution on window size $w$. Generally, we observe that for increasing $w$ the probability for long $\delta$-bursts increases, while short $\delta$-bursts become less likely (Fig. $7 b, d$, insets). When $\delta$-burst duration distributions corresponding to different window sizes $w$ are rescaled by their respective mean duration $\left\langle d_{\delta}\right\rangle$, we find that all distributions collapse onto a unique function $f_{\delta}$ following a Weibull behavior (Fig. $7 b, d$ ) and obeying the scaling relation as follows:

$$
P_{\delta}(d) \sim\left\langle d_{\delta}\right\rangle^{-\xi} \cdot f_{\delta}\left(d /\left\langle d_{\delta}\right\rangle^{\xi}\right) .
$$

As in the case of $\theta$-burst dynamics, the temporal organization of $\delta$-bursts is robust and characterized by a scaling function (Eq. 9), which is universal for the control and VLPO groups, and remains stable during light and dark periods (Figs. $8 b, d, 9 b, d$ ).

The existence of universal scaling functions (Eqs. 6-9) not only demonstrates that duration distributions are independent of the specific set of parameters used to identify $\theta$ - and $\delta$-bursts, but also constitutes evidence of scale invariance, a property associated with systems operating at criticality.

Hierarchical micro-architecture in the temporal order of quiet ( $\delta$-bursts) and active ( $\theta$-bursts) states in the sleep-wake cycle The results reported in the previous sections show a remarkable coexistence of scale-invariant power-law structure for the dura- 

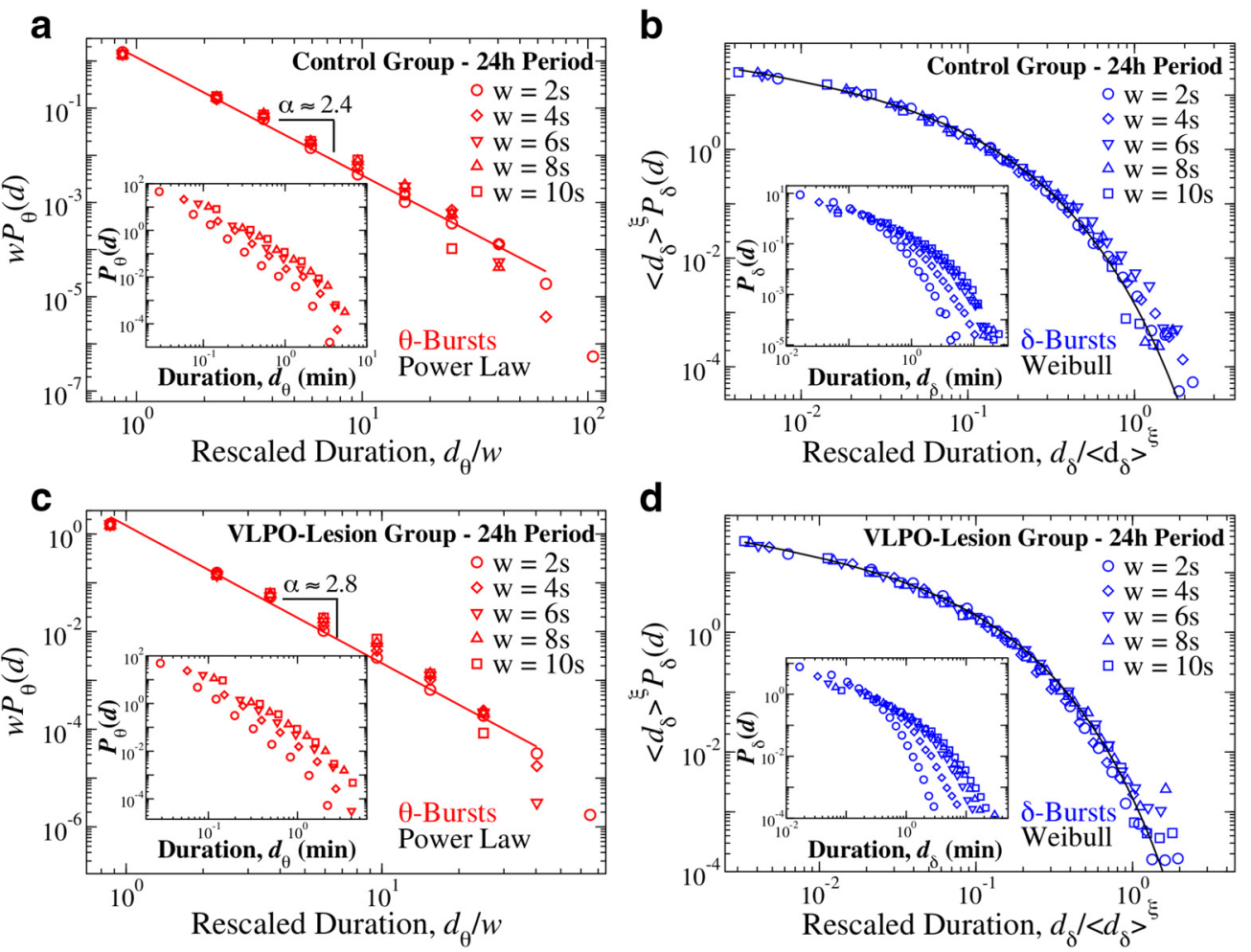

Figure 7. Critical characteristics in the dynamics of bursts of dominant cortical rhythms are independent of the scale of analysis. Distribution of $\theta$ and $\delta$-burst durations for different scales of observation defined by window sizes $w$ (Fig. 1). $\boldsymbol{a}$, Rescaled distribution of $\theta$-burst durations for control rats over a $24 \mathrm{~h}$ period (pooled data). Distributions are rescaled by the window size $w$ and consistently show the same power law behavior with $\alpha=2.4$ (red line), as proven by the data collapse. Inset, Distributions $P_{\theta}$ for different window sizes $w$ (not rescaled). $\boldsymbol{b}$, Rescaled distribution of $\delta$-burst durations for control rats over a $24 \mathrm{~h}$ period (pooled data). Distributions are rescaled by $\left\langle d_{\delta}\right\rangle^{\xi}$, where $\left\langle d_{\delta}\right\rangle$ is the mean $\delta$-burst duration and $\xi=1$, and collapse onto a single function that is well described by a Weibull distribution $f(d ; \lambda, \beta)$ with $\lambda=6.18$ and $\beta=0.72$ (black line). Inset, Distributions $P_{\delta}$ for different window sizes (not rescaled). $c$, Rescaled distribution of $\theta$ burst durations for VLP0-lesioned rats over a $24 \mathrm{~h}$ period (pooled data). Distributions are rescaled by the window size $w$ and consistently show the same power law behavior with $\alpha=2.8$ (red line), as proven by the data collapse. Inset, Distributions $P_{\theta}$ for different window sizes (not rescaled). $\boldsymbol{d}$, Rescaled distribution of $\delta$ burst durations for VLP0-lesioned rats over a $24 \mathrm{~h}$ period (pooled data). Distributions are rescaled by $\left\langle d_{\delta}\right\rangle^{\xi}$, with $\xi=1$, and collapse onto a single function following a Weibull behavior $f(d ; \lambda, \beta)$ (black line) with $\lambda=15$.20 and $\beta=0.74$. Inset, Distributions $P_{\delta}$ for different window sizes (not rescaled). Results in all panels are obtained for fixed threshold $T h=1$ on the ratio $R_{\theta \delta}$ (Fig. 1). Results are consistent when considering separately light and dark periods (Figs. 8, 9).

tions of $\theta$-bursts and a Weibull functional form with a characteristic time scale for $\delta$-burst durations (Figs. 2,3). This evidence draws a strong parallel with far-from-equilibrium phenomena that are characterized by bursting dynamics and abrupt transitions between active and quiet states, such as avalanches and earthquakes (Boffetta et al., 1999; Corral, 2004; Paczuski et al., 2005; Munoz, 2018). For instance, the intensity of avalanches and earthquakes (active states) is also described by power-law distributions, while time intervals between consecutive avalanches/ earthquakes (quiet states) follow a generalized Gamma distribution with a characteristic time scale (exponential tail). In this context Gamma is a universal scaling function that is independent of spatial scales and minimum magnitude thresholds, and is consistently observed for a broad range of conditions despite the large variability associated with phenomena such as avalanches and earthquakes (Corral, 2004; Ribeiro et al., 2010; de Arcangelis et al., 2016).

In sleep dynamics, wake and brief arousals during sleep can be considered as active states that, in rodents, are characterized by bursts in $\theta$ rhythms. Hence, we hypothesize that a hierarchical structure, invariant across time scales, may underlie the occurrence of $\theta$-bursts, in analogy with non-equilibrium critical phenomena, and investigate the relationship between the duration of $\theta$-bursts and their temporal occurrence (Fig. 10). To this end, we consider the time sequence of $\theta$-bursts, and we study the statisti- cal features of the quiet times $\Delta t$ separating consecutive bursts, taking into account the duration $d_{\theta}$ of each $\theta$-burst (Fig. 10a). Because $\theta$-bursts vary in duration, we impose a threshold $D_{0}$ representing the time scale of analysis, and we define the quiet time $\Delta t_{i}$ as the period from the end of $\theta_{i}$-burst to the beginning $\theta_{i+1}$-burst. Thus, the statistical characteristics of $\Delta t_{i}$ depend on the threshold value $D_{0}$. We then obtain the probability distribution $P\left(\Delta t ; D_{0}\right)$ of quiet times $\Delta t_{i}$ for different values of $D_{0}$ (Fig. $6 c, d$, insets). With increasing threshold (scale of observation) $D_{0}$, the probability of longer $\Delta t_{i}$ increases, whereas the probability of short $\Delta t_{i}$ decreases, leading to different curves for the distributions $P\left(\Delta t ; D_{0}\right)$.

Visual inspection of the complex profile formed by the time sequence of $\theta$-bursts and their respective durations shows an apparent similarity when comparing short segments of the profile with the entire sequence above a given threshold $D_{0}$ (Fig. 6b). Presence of statistical self-similarity, observed after effective coarse-graining of the profile, indicates a hierarchical structure across time scales $D_{0}$ that characterizes $\theta$-bursts occurrence times and durations, and the associated quiet times. To demonstrate statistical self-similarity in the quiet times, we systematically analyze the functional form of the probability distributions $P\left(\Delta t ; D_{0}\right)$ for different thresholds $D_{0}$ by rescaling each distribution by the average quiet time $\langle\Delta t\rangle_{D_{0}}$. Remarkably, we find that all distribu- 

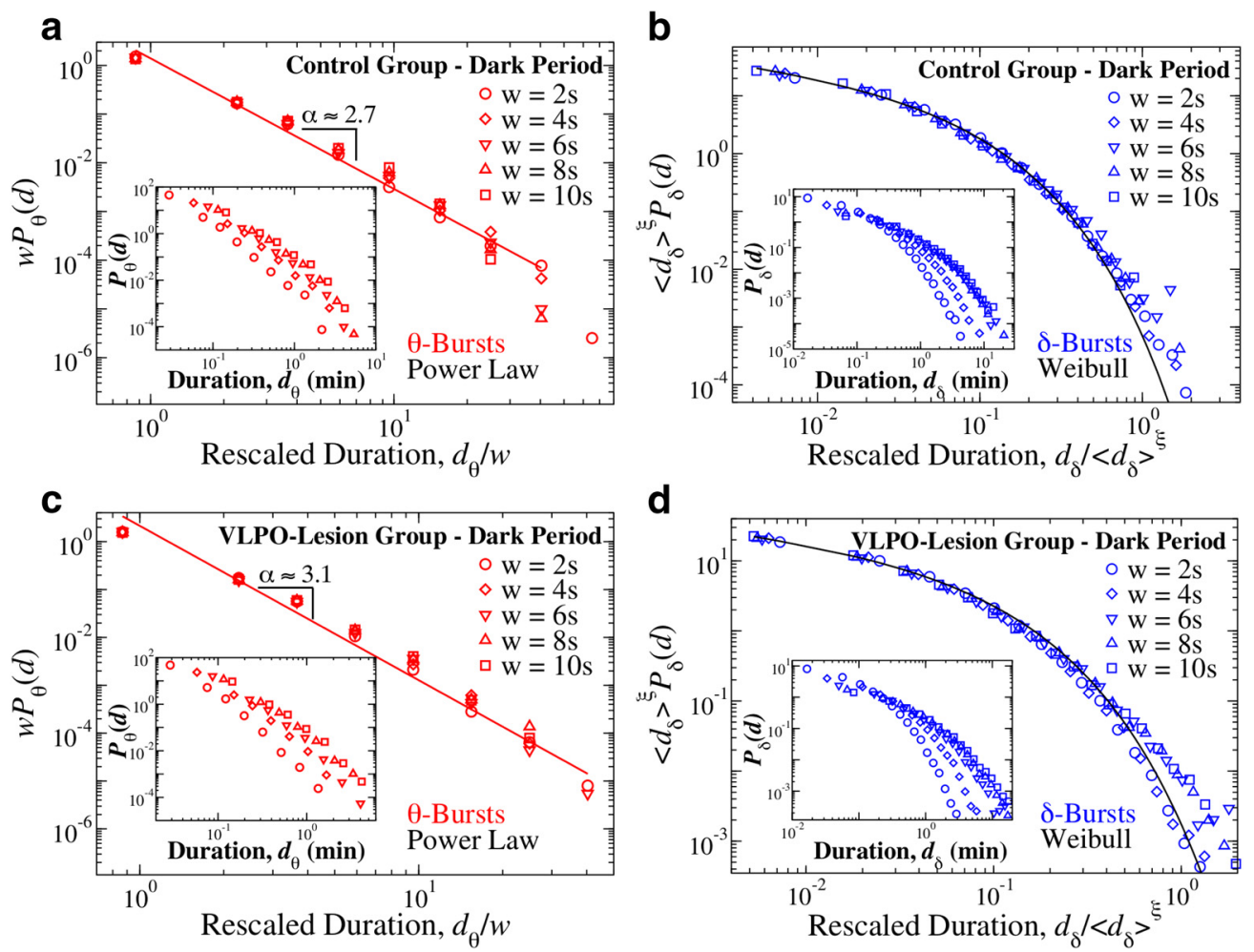

Figure 8. Distribution of $\theta$ - and $\delta$-burst durations in the dark period are independent of the scale of analysis and can be described by unique scaling functions. Distribution of $\theta$ and $\delta$ burst durations in dark period for different window sizes $w$ and $T h=1$ on the ratio $R_{\theta \delta} \cdot \boldsymbol{a}$, Rescaled distribution of $\theta$ burst durations for control rats over a $12 \mathrm{~h}$ dark period (pooled data). Distributions are rescaled by the window size $w$ and consistently show the same power law behavior with $\alpha=2.65$, as proven by the data collapse. Inset, Distributions $P_{\theta}$ for different window sizes (not rescaled). $\boldsymbol{b}$, Rescaled distribution of $\delta$ burst durations for control rats over a $12 \mathrm{~h}$ dark period (pooled data). Distributions are rescaled by $\left\langle d_{\delta}\right\rangle^{\xi}$, with $\left\langle d_{\delta}\right\rangle$ mean $\delta$-burst duration and $\xi=1$, and collapse onto a signle function $f_{\delta}$ that is well described by a Weibull distribution $f(x ; \lambda, \beta)$ (black line), with $\beta=0.66$ and $\lambda=0.04$. Inset, Distributions $P_{\delta}$ for different window sizes (not rescaled). $c$, Rescaled distribution of $\theta$ burst durations for VLPO lesioned rats over a $12 \mathrm{~h}$ dark period (pooled data). Distributions are rescaled by the window size $w$ and consistently show the same power law behavior with $\alpha=3.1$, as proven by the data collapse. Inset, Distributions $P_{\theta}$ for different window sizes (not rescaled). $\boldsymbol{d}$, Rescaled distribution of $\delta$ burst durations for VLP0-lesion rats over a $12 \mathrm{~h}$ dark period (pooled data). Distributions are rescaled by $\left\langle d_{\delta}\right\rangle^{\xi}$, and collapse onto a single function that is well fitted by a Weibull distribution $f(x ; \lambda, \beta)$ (black line), with $\beta=0.70$ and $\lambda=0.05$. Inset, Distributions $P_{\delta}$ for different window sizes (not rescaled).

tion curves collapse onto a single function $G$ (Fig. $6 c, d$ ), defined by the following scaling relation:

$$
P(\Delta t)=\langle\Delta t\rangle^{-1} \cdot G(\Delta t /\langle\Delta t\rangle) .
$$

The scaling relation in Equation 10 represents a mathematical expression of the statistical self-similarity in the profile formed by the quiet times and $\theta$-burst durations shown in Figure $6 b$. We find that the scaling function $G(\Delta t /\langle\Delta t\rangle)$ is well described by the generalized Gamma distribution $G(\Delta t /\langle\Delta t\rangle ; b, v, p)=p / b^{v}(\Delta t /\langle t\rangle)^{v-1} e^{-(\Delta t / b(t) p} / \Gamma(v / p)($ Stacy, 1962), where in our analysis $\Delta t /\langle\Delta t\rangle$ is a dimensionless quiet time. The Gamma functional form is homogeneous (Ivanov et al., 1996) i.e., rescaling the variable leaves the functional form unchanged. Such scaling function indicates a hierarchical structure in the quiet times between consecutive $\theta$-bursts, independent of the scale of observation $D_{0}$. In the limit of $D_{0}=0$, the quiet time distribution $P\left(\Delta t ; D_{0}\right)$ coincides with the distribution of $\delta$-burst durations $P_{\delta}$ (Figs. $2 b, 3 b, d$ ); a Weibull functional form that belongs to the same class of homogeneous functions as the generalized Gamma.

Our analysis shows that the scaling relation in Equation 10 and the associated Gamma functional form for the quiet times are robust: (1) we find them in the $24 \mathrm{~h}$ period (Fig. 10) as well as separately during light and dark periods, and (2) they do not significantly change with lesion in the VLPO (Fig. 10, compare $c$, d). Further, the presence of such hierarchical structure in quiet times indicates specific temporal order in the occurrence of $\theta$-bursts. To explicitly verify this, we randomly reshuffle the sequence of $\theta$-burst durations, while preserving the $\delta$-burst durations corresponding to quiet times at $D_{0}=0$, and we perform the analysis on the reshuffled sequence to obtain quiet time distributions $P_{\text {rand }}\left(\Delta t ; D_{0}\right)$ for different thresholds $D_{0}$. In this case, after rescaling the distributions $P_{\text {rand }}\left(\Delta t ; D_{0}\right)$ by the average quiet time $\langle\Delta t\rangle_{D_{0}}$, their curves collapse onto an exponential distribution (Fig. 10c,d, dashed lines); a hallmark of temporal independence between consecutive events (Daley and Vere-Jones, 1988). This clearly demonstrates that temporal correlations are intimately related to the existence of non-exponential scaling functions (Eq. 10; Daley and Vere-Jones, 1988; Corral, 2004).

Notably, a similar temporal organization characterized by coexistence of power-law and generalized Gamma distribution has been reported for active states and quiet times in a range of nonequilibrium systems self-tuning at criticality (earthquakes, avalanches; Corral, 2004; Pruessner, 2012; Munoz, 2018). Thus, our findings of power-law distribution for $\theta$-burst durations (Figs. 2,3 ) combined with a generalized Gamma distribution for the quiet times between consecutive $\theta$-bursts at different scales of observation $D_{0}$ (Fig. 10) are a strong evidence in support of our hypothesis that bursting activity of fundamental brain rhythms and the associated sleep micro-architecture exhibit critical nonequilibrium behavior. 

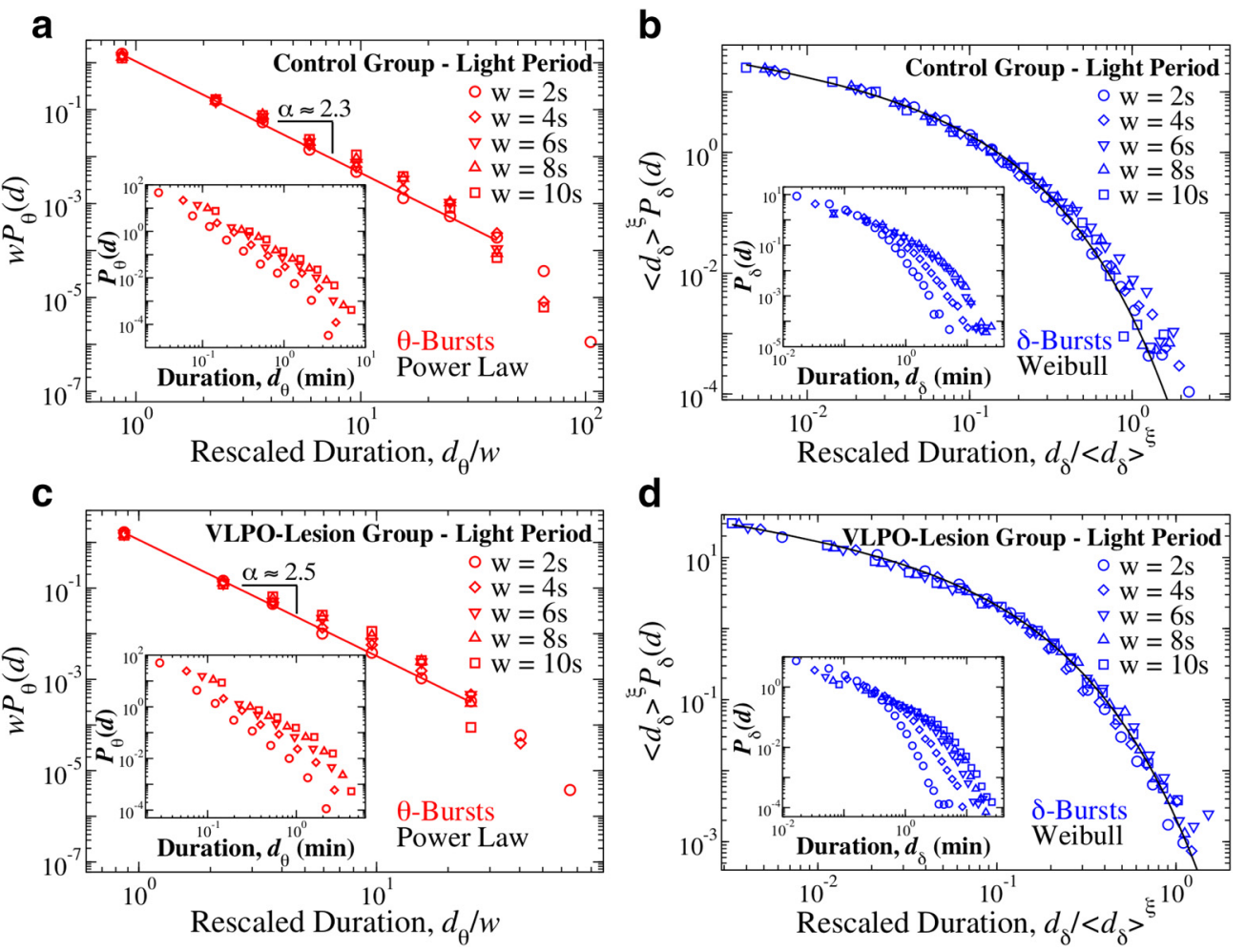

Figure 9. Distribution of $\theta$ - and $\delta$-burst durations in the light period are independent of the scale of analysis and can be described by unique scaling functions. Distribution of $\theta$ and $\delta$ burst durations in light period for different window sizes $w$ and $T h=1$ on the ratio $R_{\theta \delta}$. $\boldsymbol{a}$, Rescaled distribution of $\theta$ burst durations for control rats over a $12 \mathrm{~h}$ light period (pooled data). Distributions are rescaled by the window size $w$ and consistently show the same power law behavior with $\alpha=2.3$, as proven by the data collapse. Inset, Distributions $P_{\theta}$ for different window sizes (not rescaled). $\boldsymbol{b}$, Rescaled distribution of $\delta$ burst durations for control rats over a $12 \mathrm{~h}$ light period (pooled data). Distributions are rescaled by $\left\langle d_{\delta}\right\rangle^{\xi}$, with $\left\langle d_{\delta}\right\rangle$ mean $\delta$-burst duration and $\xi=1$, and collapse onto a single function $f_{\delta}$ that is well described by a Weibull distribution $f(x ; \lambda, \beta)$ (thick black line), with $\lambda=0.04$ and $\beta=0.70$. Inset, Distributions $P_{\delta}$ for different window sizes (not rescaled). c, Rescaled distribution of $\theta$ burst durations for VLPO lesioned rats over a $12 \mathrm{~h}$ light period (pooled data). Distributions are rescaled by the window size $w$ and consistently show the same power law behavior with $\alpha=2.5$, as proven by the data collapse. Inset, Distributions $P_{\theta}$ for different window sizes (not rescaled). $\boldsymbol{d}$, Rescaled distribution of $\delta$ burst durations for VLPO-lesion rats over a $12 \mathrm{~h}$ light period (pooled data). Distributions are rescaled by $\left\langle d_{\delta}\right\rangle^{\xi}$, with $\xi=1$, and collapse onto a single function $f_{\delta}$ that is well described by a Weibull distribution $f(x ; \lambda, \beta)$ (thick black line), with $\lambda=0.04$ and $\beta=0.67$. Inset, Distributions $P_{\delta}$ for different window sizes (not rescaled).

\section{Long-range power-law correlations in the durations of $\theta$ and $\delta$ bursts}

Physical systems at criticality exhibit long-range correlations that span the entire system across space and time scales, and lead to the emergence of collective cooperative behavior (Stanley, 1987). Indeed, scaling features in such systems often arise in conjunction with long-range spatiotemporal correlations following power-laws. Notably, physiological systems under neuro-autonomic regulation also exhibit dynamics characterized by long-range power-law correlations; a scale-invariant structure that undergoes a phase transition with transitions from sleep to wake (Kantelhardt et al., 2003; Schmitt et al., 2009), with circadian rhythms (Hu et al., 2004; Ivanov, 2007) and under clinical conditions (Ivanov et al., 1999; Goldberger et al., 2002). Further, the randomization procedure in the previous subsection (Fig. 10) clearly demonstrates that the scale invariant structure in quiet times characterized by a Gamma scaling function (Eq. 10) can arise only in the presence of a certain temporal order in $\theta$-bursts occurrence. Thus, we next perform correlation analysis to quantify long-range features in the temporal organization of $\delta$ - and $\theta$-burst durations.

To this end, we use the DFA, a random walk based method specially tailored to quantify long-range power-law correlations embedded in nonstationary signals with various polynomial trends and bursting dynamics (Chen et al., 2002, 2005). The DFA method is based on evaluation of the rms fluctuation function $F(n)$ (see Materials and Methods, Data analysis), where $n$ is the scale of analysis expressed in number of consecutive bursts (Fig. 11). A scaling relationship of the form $F(n) \propto n^{\alpha_{d}}$ indicates presence of long-range power-law correlations in the time series of burst durations. Correlation exponent $\alpha_{d} \in(0,0.5)$ indicates anti-correlations (where short burst durations tend to be followed by longer burst durations), whereas $\alpha_{d} \in(0.5,1)$ indicates positive persistent correlations (long bursts tend to be followed by longer bursts); $\alpha_{\mathrm{d}}=0.5$ corresponds to a random walk and absence of correlations.

We perform DFA on sequences of $\theta$ - and $\delta$-burst durations separately, distinguishing between dark and light periods (Fig. $11)$. We find that both $\theta$ - and $\delta$-bursts exhibit long-range powerlaw correlations. In control rats, the power-law exponents characterizing $\theta$ - and $\delta$-bursts are $\alpha_{\mathrm{d}}=0.58 \pm 0.02$ and $\alpha_{\mathrm{d}}=0.64 \pm$ 0.01 (exponent \pm error on fit) for the $12 \mathrm{~h}$ dark period, and $\alpha_{\mathrm{d}}=$ $0.61 \pm 0.01$ and $\alpha_{\mathrm{d}}=0.64 \pm 0.01$ (exponent \pm error on fit) for the $12 \mathrm{~h}$ light period. Comparisons between group averages show that exponents for dark and light periods are not significantly different [ $\theta$-bursts: Control dark $\alpha_{\mathrm{d}}=0.57 \pm 0.05$ vs Control light $\alpha_{\mathrm{d}}=0.56 \pm 0.04$ (mean $\pm \mathrm{SD}$ ), $t$ test, $p=0.636$; $\delta$-bursts: Control dark $\alpha_{\mathrm{d}}=0.61 \pm 0.07$ vs Control light $\alpha_{\mathrm{d}}=0.59 \pm 0.04$ (mean $\pm \mathrm{SD}$ ), Mann-Whitney $U$ test, $p=0.751]$. Moreover, our analysis indicates that VLPO lesions do not affect the nature of temporal correlations, but may influence their strength (Fig. 11). As for control rats, power-law exponents characterizing correlations during dark and light periods are not significantly different 

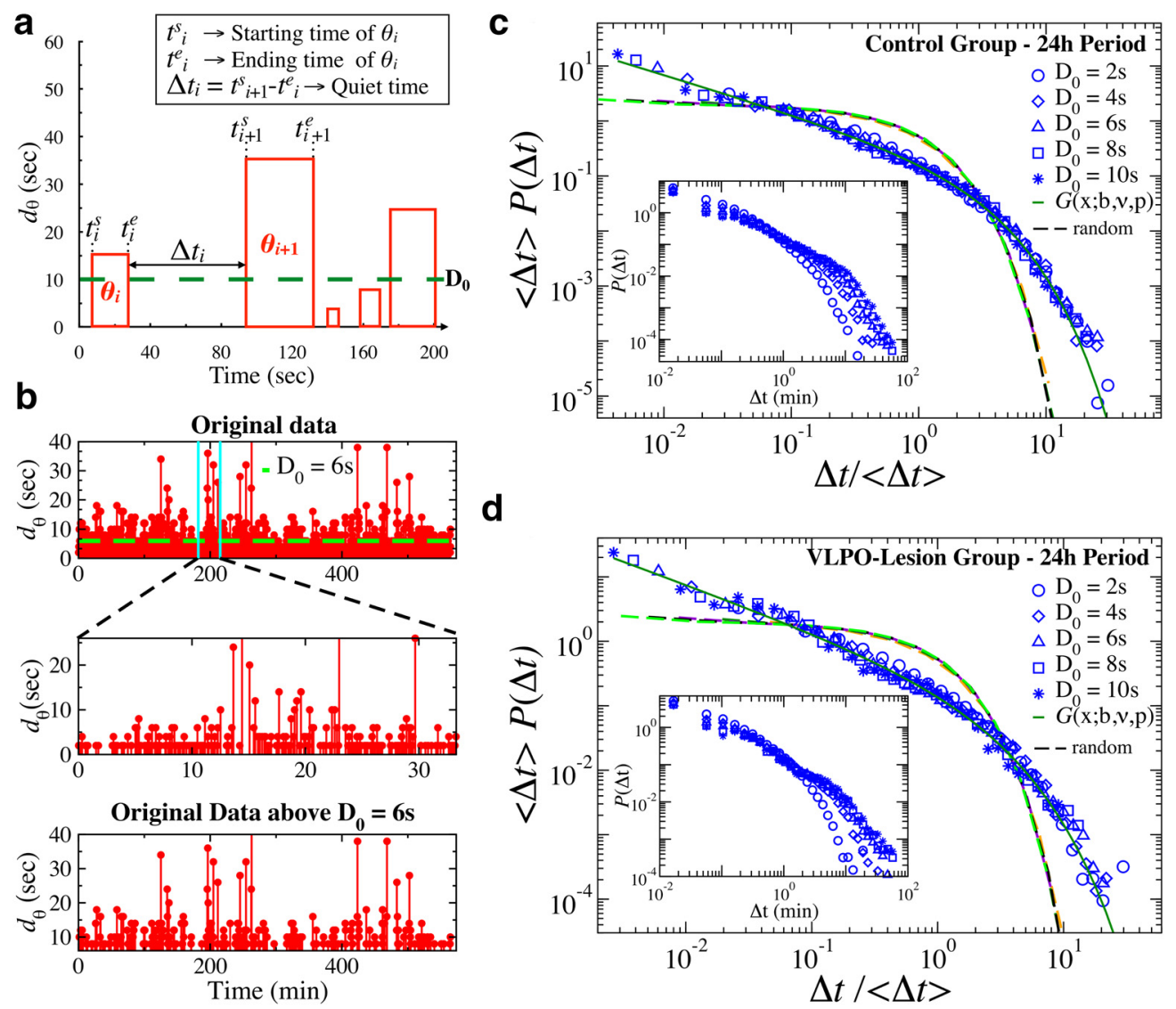

Figure 10. Hierarchical self-similar structure in quiet times between consecutive $\theta$-bursts indicates coupling between time of occurrence and burst duration. $\boldsymbol{a}$, Schematic diagram of quiet times $\Delta t$ between consecutive $\theta$-bursts. A quiet time $\Delta t_{i}$ is the time elapsed from the end of burst $\theta_{i}$ to the beginning of the following burst $\theta_{i+1} \cdot \boldsymbol{b}$, Top, Time series of $\theta$-burst durations for $\sim 600$ min recording of a control rat. Middle, A 40 min segment from the sequence shown at the top. Bottom, Sequence comprised only of the $\theta$-burst durations longer than $D_{0}=6 \mathrm{~s}$ that are present in the 600 min time series shown at the top. Selecting only bursts longer than $D_{0}=6 \mathrm{~s}$, the temporal pattern at the scale of 600 min looks similar to the pattern at smaller scale of 60 min, indicating hierarchical self-similar structure in the quiet times. c, Distribution of quiet times for different thresholds $D_{0}$ on $\theta$-burst durations over a $24 \mathrm{~h}$ period in control rats (blue symbols). When rescaled by $\langle\Delta t\rangle$ (main panel), distributions obtained for different $D_{0}$ collapse onto a unique function that is well described by a generalized Gamma distribution $G(x ; b, v, p)$ (solid green line), with $b=2.03, \nu=0.30$, and $p=0.81$. Applying the same procedure to a sequence of randomly reshuffled $\theta$-burst durations leads to distributions that collapse onto an exponential function (dashed lines). Inset, Distributions of quiet times for different thresholds $D_{0}$ before rescaling. $\boldsymbol{d}$, Distributions of quiet times for different thresholds $D_{0}$ on $\theta$-burst durations over a $24 \mathrm{~h}$ period in VLPO-lesioned rats. Distributions collapse onto a unique function when rescaled by $\langle\Delta t\rangle$ (main panel). Similar to control rats, this function is well described by a generalized Gamma function $G(x ; b, v, p)$ (solid green line), with $b=1.55, \nu=0.28$, and $p=0.70$. Distribution of quiet times obtained from a sequence of randomly reshuffled $\theta$-burst durations collapse onto an exponential distribution (dashed lines). Insets, Distributions of quiet times in VLP0-lesioned rats for different thresholds $D_{0}$ before rescaling. Results are consistent when considering separately light and dark periods.

[ $\theta$-bursts: VLPO dark $\alpha_{\mathrm{d}}=0.57 \pm 0.07$ vs VLPO light $\alpha_{\mathrm{d}}=$ $0.58 \pm 0.08$ (mean $\pm \mathrm{SD}$ ), $t$ test, $p=0.839 ; \delta$-bursts: VLPO dark $\alpha_{\mathrm{d}}=0.57 \pm 0.04$ vs VLPO light $\alpha_{\mathrm{d}}=0.57 \pm 0.03$ (mean $\pm \mathrm{SD}$ ), $t$ test, $p=0.986$ ]. Comparison between group averages do not show significant differences between DFA $\theta$-burst exponents in control and VLPO-lesioned rats (Control dark vs VLPO dark, $t$ test, $p=0.916$; Control light vs VLPO light, $t$ test, $p=0.475$ ). Differences appear more pronounced for the DFA $\delta$-burst exponents, with the VLPO exponent $\alpha_{\mathrm{d}}=0.59 \pm 0.01$ lower than the control exponent $\alpha_{\mathrm{d}}=0.64 \pm 0.01$. However, the comparison between control and the VLPO-lesioned group do not evidence significant differences (Control dark vs VLPO dark, Mann-Whitney $U$ test, $p=0.436$; Control light vs VLPO light, $t$ test, $p=0.190$ ).

\section{Anti-correlated coupling between the durations of consecutive $\boldsymbol{\theta}$ - and $\boldsymbol{\delta}$-bursts}

Physical and biological systems at equilibrium (homoeostasis) are typically controlled by mechanisms that either lead to dynamics with specific space or time scales characterized by exponential behaviors or to scale-invariant dynamics without characteris- tic scales following power-laws. Non-equilibrium systems selforganizing at criticality are unique in the sense that they combine two distinct behaviors, a scale-invariant power-law related to the dynamics of active states and an exponential related to quiet states, both of which emerge out of a single regulatory mechanism (Lo et al., 2002). In that context, our findings of (1) power-law distribution for $\theta$-burst (active states) durations in coexistence with Weibull distribution for $\delta$-burst (quiet states) durations (characteristic time scale in the quiet states) shown in Figures 2 and 3, (2) universal Gamma function characterizing the temporal organization of quiet times across a range of scales (Fig. 10), and (2) long-range power-law correlations in the time sequence of $\theta$ and $\delta$-burst durations (Fig. 11), all typical features of systems at criticality, indicate a common regulatory mechanism for the bursting activity of both $\theta$ and $\delta$ rhythms and associated sleep micro-architecture. Presence of such common mechanism may imply coupling between $\theta$ - and $\delta$-bursts, as further suggested by concurrent change in both power-law and Weibull distribution parameters with transition from dark to light periods (Fig. 3). Thus, to further understand bursting dynamics in relation to 

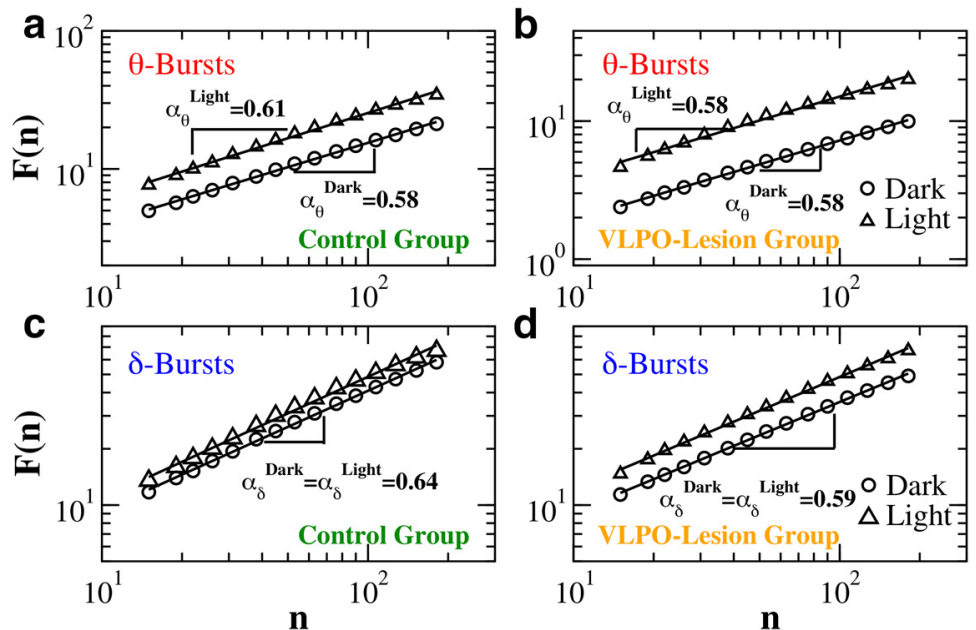

Figure 11. Long-range power-law correlations in sequences of consecutive $\theta$ - and $\delta$-burst durations indicate a dynamical system at criticality. Detrended fluctuation analysis for sequences of $\theta$ - and $\delta$-burst durations in control and VLPO-lesioned rats. Burst durations are calculated using a window $w=4$ s and threshold Th $=1$ on the ratio $R_{\theta \delta}$ (Fig. 1), and are analyzed separately for $12 \mathrm{~h}$ dark/light periods. The rms fluctuation function $F(n)$ is obtained averaging over all rats in the control $(\boldsymbol{a})$ and VLPO-lesioned group $(\boldsymbol{b})$, respectively. $\log -\log$ plots of $F(n)$ versus the time scale of analysis $n$, where $n$ is the number of consecutive burst durations, show power-law relations $F(n) \propto n^{\alpha_{d}}$ over a broad range of scales $n$. The scaling exponents are significantly larger than 0.5 , both in light and dark periods, indicating presence of positive (persistent) long-range correlations in $\theta$-bursts for both control and VLPO-lesioned rats. Similar results are found in sequences of $\delta$-bursts for $(\boldsymbol{c})$ control and (d) VLP0-lesioned rats. Power-law exponents were obtained from the average rms fluctuation function $F(n)$.

neuronal integrity in the VLPO, we next investigate the coupling between consecutive $\delta$ - and $\theta$-burst durations, and the role of such coupling in the emergent scaling behavior of duration distributions in control and VLPO-lesioned rats.

We first focus on the relationship between ranks of consecutive $\delta$ - and $\theta$-burst durations, $d_{\delta}$ and $d_{\theta}$. We rank burst durations in ascending order, with the shortest duration corresponding to the smallest rank, and examine the scatter plots between the ranks of consecutive $d_{\delta}$ and $d_{\theta}$ (Fig. 12a,b). We find that $\delta$-bursts of high ranks (i.e., long durations) tend to be followed by $\theta$-bursts of low ranks (i.e., short durations). This anti-correlated coupling is consistently present in both control (Fig. 12a) and VLPOlesioned rats (Fig. 12b), and appears to be a basic characteristic of dynamics as it is observed throughout the entire sleep-wake cycle in both dark and light periods.

To quantify the coupling between consecutive $\delta$ - and $\theta$-burst durations we use Spearman's correlation coefficient, which assesses monotonic relationships between two variables (see Materials and Methods, Data analysis). The Spearman's crosscorrelation is positive when observations of two variables have similar ranks, and negative if they have opposite ranks. Our analyses show that the Spearman's coefficient calculated for consecutive $\delta$ - and $\theta$-burst durations is always ( $24 \mathrm{~h}$, dark/light period) significantly negative (Fig. 12c), indicating anti-correlated coupling. This is verified by a surrogate test where the sequence of consecutive $\delta$ - and $\theta$-burst durations is randomized (Fig. 12c; see Materials and Methods, Data analysis). We find that the $\delta-\theta$ anti-correlated coupling is less pronounced for VLPO-lesioned rats, and this is consistently observed in both light and dark periods. Comparing light versus dark period, our results show an increase in the anti-correlated coupling during the light period within each group (Fig. 12c).

The presence of anti-correlated coupling between consecutive $\delta$ and $\theta$-bursts is further supported by the analysis of conditional probabilities (Fig. 13). Specifically, we ask how the conditional probability distribution $P\left(d_{\delta_{i}} \mid d_{\theta_{i-1}}>d^{*}\right)$ of $\delta$-burst durations $d_{\delta_{i}}$ changes depending on the length of preceding $\theta$-burst duration $d_{\theta_{i-1}}$, i.e., we consider the probability distribution of the subset of $\delta$-bursts which follow $\theta$-burst durations above a given length $d^{*}$. We note that if $d_{\delta_{i}}$ is independent of the preceding $\theta$-burst, then the conditional probability distribution is equivalent to the unconditioned Weibull distribution of $\delta$-burst durations (Fig. 3), i.e., $P\left(d_{\delta_{i}} \mid d_{\theta_{i-1}}>d^{*}\right)=P\left(d_{\delta_{i}}\right)$. On the other hand, finding $P\left(d_{\delta_{i}} \mid d_{\theta_{i-1}}>d^{*}\right) \neq P\left(d_{\delta_{i}}\right)$ implies that the duration $d_{\delta_{i}}$ depends on the duration $d_{\theta_{i-1}}$ of the preceding $\theta$-burst. By studying the behavior of the conditional probability distribution $P\left(d_{\delta_{i}} \mid d_{\theta_{i-1}}>d^{*}\right)$ for several values of the threshold $d^{*}$, we find that the probability for longer $d_{\delta_{i}}$ systematically drops for increasing $d^{*}$ values (faster declining tail of the conditional distribution), while the probability for shorter $d_{\delta_{i}}$ significantly increases (Fig. 13, insets). This dependence is observed during light and dark periods for both control and VLPO-lesioned groups, and indicates an anti-correlated coupling between the durations of consecutive $\theta$ - and $\delta$-bursts.

\section{Phenomenological model of coupling and criticality in $\theta$ - and $\delta$-bursts dynamics}

We next test whether the established anti-correlated coupling between consecutive $\delta$ - and $\theta$-burst durations is essential for the emergent duality of power-law and Weibull distribution. We develop a phenomenological model (Fig. 14) based on anticorrelated pairing of $\theta$ and $\delta$ durations randomly drawn from the empirical distributions of the $\delta$ - and $\theta$-burst durations established in our study (Fig. 2). The model allows to control the degree of anti-correlations between consecutive $\theta$ - and $\delta$-burst durations, and thus to examine whether and how anti-correlations affect the emerging power-law and Weibull distributions of burst durations, and the related scale-invariant temporal structure of $\theta$ - and $\delta$-bursts.

The basic steps to generate sequences of alternating $\theta$ - and $\delta$-burst durations with the desired degree of correlations are schematically outlined in Figure 14 and explained in further detail in Materials and Methods. Specifically: (1) we randomly draw durations $d_{\theta}$ and $d_{\delta}$ from their respective power-law and Weibull distributions obtained from our empirical data analyses (Fig. $15 a) ;(2) d_{\theta}$ and $d_{\delta}$ are next separately sorted in ascending order, from shortest to longest, and are assigned a unique rank (Fig. 15b); (3) the durations $d_{\theta}$ and $d_{\delta}$ are then paired based on their ranks with a certain degree of anti-correlation (defined by and monotonically depending on a single parameter) to generate an artificial time series of alternating $\theta$ - and $\delta$-burst durations (Fig. 15b); and (4) the obtained artificial time series is then binarized in windows $w$ corresponding to the window size used in our EEG spectral power analysis of the original data (Fig. 1a), where + is assigned for $\theta$-bursts and - for $\delta$-bursts. Finally, the binary series is coarse-grained at larger time scale $\Delta$ (Fig. 14c).

We use this model to test our hypothesis that coupling between $\delta$ - and $\theta$-bursts is essential for the emergent duality of power-law and Weibull behavior across time scales. We test to what extent $\delta-\theta$ coupling strength plays role in the emergent 

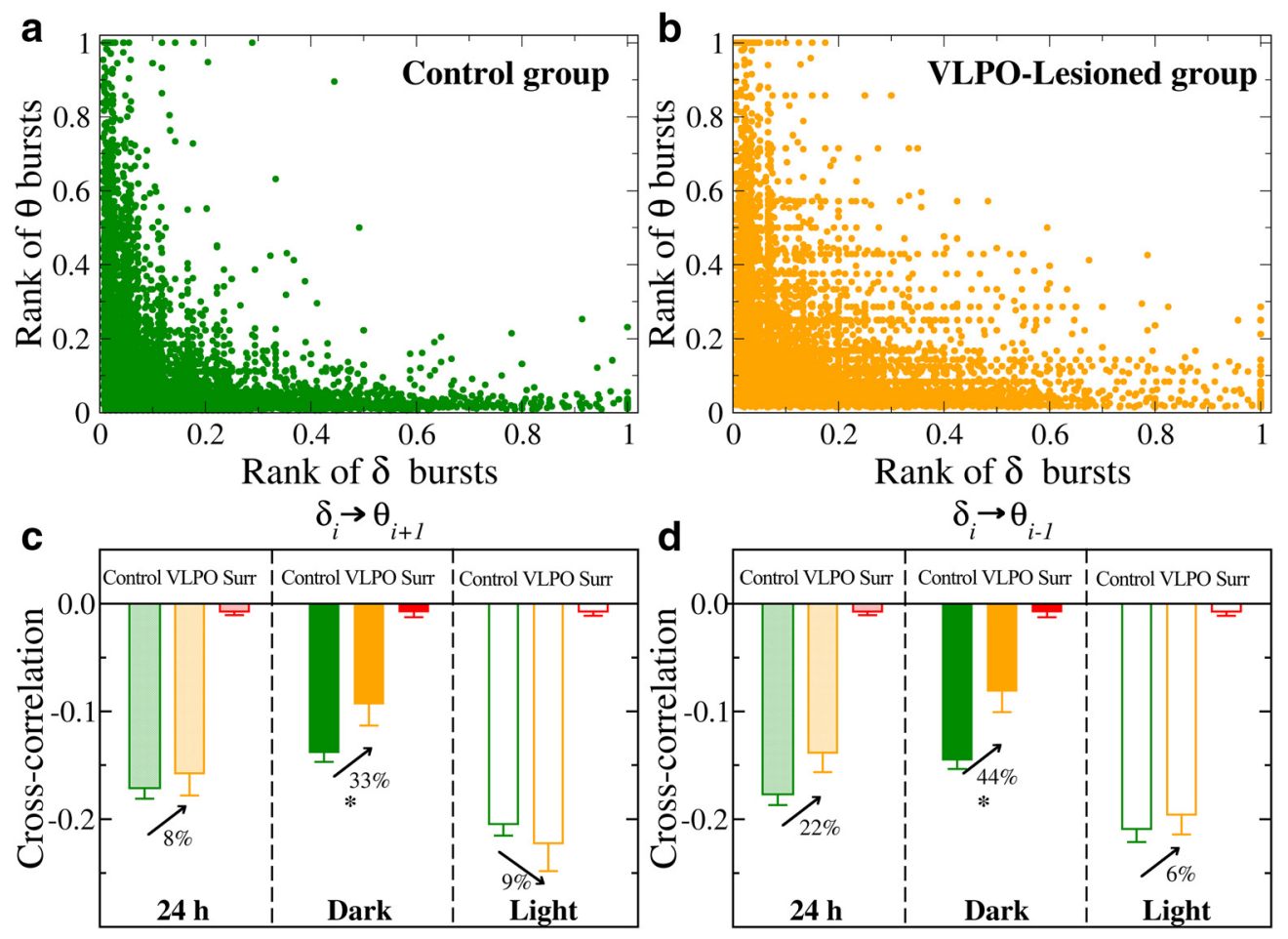

Figure 12. Coupling between $\delta$ - and $\theta$-burst durations indicates a common mechanism regulating the activity of these rhythms across the sleep-wake cycle. Scatter plots and rank correlation analysis demonstrate anti-correlated coupling between consecutive $\delta$ - and $\theta$-burst durations. $\boldsymbol{a}$, Scatter plot of $\delta$ burst ranks versus $\theta$ burst ranks in the dark period for control rats. Each dot represents a pair formed by a $\delta$-burst and the following $\theta$-burst, with burst durations separately ranked among the $\delta$-bursts and the $\theta$-bursts (longest duration corresponding to highest rank). $\boldsymbol{b}$, Scatter plot of $\delta$-burst ranks versus $\theta$-burst ranks in the dark period for VLPO-lesioned rats. For each rat group, ranks are calculated separately for each rat and then plotted together. $\boldsymbol{c}, \boldsymbol{d}$, Average Spearman's cross-correlation coefficient for control and VLPO-lesioned rats in dark, light, and $24 \mathrm{~h}$ periods. Anti-correlations between consecutive $\theta$-and $\delta$-bursts are stronger during light than during dark periods in each group. Comparing dark versus light periods, the Student's $t$ test gives $p<0.001$ for control rats and $p=0.002$ for VLPO-lesioned rats. Importantly, VLPO lesioned rats generally exhibit weaker anti-correlations than the control group, in particular during dark periods, where anti-correlation decreases with $\approx 35 \%$ compared with control rats [Control vs VLPO $t$ test; (c) $24 \mathrm{~h}, p=0.159 ;$ dark, $p=0.008$; light, $p=0.700 ;$ (d) $24 \mathrm{~h}, p=0.069 ;$ dark, $p=0.013$; light, $p=0.273$ ]. All correlation coefficients calculated in both groups are significantly different from the corresponding values obtained in the surrogates (red bars) after randomly reshuffling the original order of $\theta$-and $\delta$-bursts $(t$ test, $p<0.001)$. All durations are calculated using a window $w=$ 4 s and threshold $T h=1$ on the ratio $R_{\theta \delta}$ (as in Fig. 1).

scale-invariant organization of sleep micro-architecture. Our simulations show that the generated distributions of $\delta$ - and $\theta$-burst durations depend on the degree of anti-correlation introduced in the model. When the Spearman's cross-correlation coefficient of burst durations generated by the model corresponds to the empirical values found in real data, the distributions obtained from the model approximate the empirical distributions (Fig. 15), and scale-invariant temporal organization in burst durations emerges over a range of coarse-graining scales $\Delta$. In contrast, absence of anti-correlated coupling in our model (i.e., random pairing of $\delta$ and $\theta$ durations in the generated time series) leads to exponential distribution for both $\delta$ - and $\theta$-bursts, significantly different from real data (Fig. 15).

\section{Discussion}

We studied the dynamical features of wake- and sleep-dominant brain rhythms across $48 \mathrm{~h}$ recordings of the sleep-wake cycle. To uncover basic principles underlying sleep dynamics and emergent sleep stage and arousals/wake transitions, we analyzed control rats and rats with lesions in the VLPO (Lu et al., 2000, 2002). We found that transient bursts in $\theta$ and $\delta$ cortical rhythms continuously occur during the sleep-wake cycle, and exhibit a complex temporal organization which is characterized by a remarkable duality of scale-invariant power-law distribution for $\theta$-burst durations (active states) and Weibull distribution with a exponential characteristic time scale for $\delta$-burst durations (quiet states; Fig. 2). Further, we showed the presence of anti-correlated coupling between $\theta$ - and $\delta$-bursts dynamics (Fig. 12) and demonstrated that this coupling is essential part of the mechanism responsible for the emerging duality of power-law and Weibull behavior across time scales (Figs. 14, 15). The presence of complex temporal organization and coupling in cortical rhythms is also manifested through the hierarchical structure in the quiet time intervals separating consecutive active states $(\theta$ bursts) above a given duration (Fig. 10), which we find to be described by a unique scaling function (generalized Gamma distribution). This self-similar structure links, across time scales, the duration of a given $\theta$-burst with the time of its occurrence. Moreover, we found that sequences of consecutive $\theta$ - or $\delta$-burst durations are long-range power-law correlated, indicating a scale-invariant organization in the temporal order of burst durations and a unique underlying process with persistent "memory" spanning over a wide range of scales that statistically couples the duration of a given burst with the durations of hundreds of following bursts (Fig. 11).

Importantly, our empirical analyses showed that the reported characteristics of $\theta$ - and $\delta$-bursts dynamics do not depend on the scale of observation or on the threshold used to identify $\theta$ - from $\delta$-bursts, and remain continuously present during dark and light periods (Figs. 4-9), under different dominant physiologic states (sleep and wake), and both in control and VLPO-lesioned rats. The presence of multiple scale-invariant characteristics related to distributions, correlations, coupling, and timing of bursting events, 

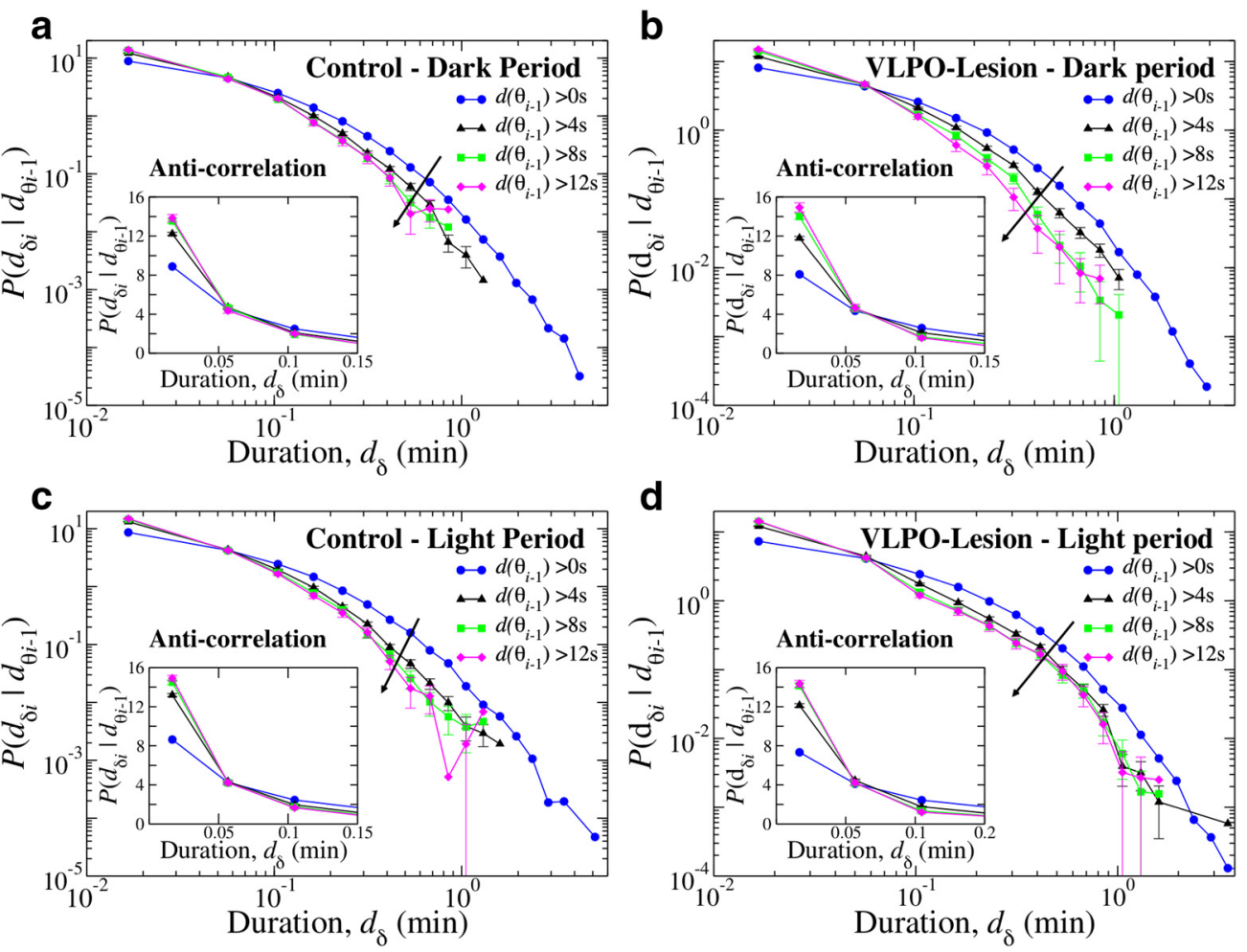

Figure 13. Distributions of conditional probabilities for $\delta$-burst durations show anti-correlated coupling between $\theta$-and $\delta$-bursts. $\boldsymbol{a}$, Distribution of durations $d_{\delta_{i}}$ given that the duration of the preceding $\theta$-burst is larger than a given threshold $d^{*}$ for control rats in $12 \mathrm{~h}$ light periods. $\boldsymbol{b}$, Distribution of durations $d_{\delta_{i}}$ given that the duration of the preceding $\theta$-burst is larger than a given threshold $d^{*}$ for VLPO-lesioned rats in $12 \mathrm{~h}$ light periods. $c$, Distribution of durations $d_{\delta_{i}}$ given that the duration of the preceding $\delta$-burst is larger than a threshold $d^{*}$ for control rats in $12 \mathrm{~h}$ dark period. $\boldsymbol{d}$, Distribution of durations $d_{\delta_{i}}$ given that the duration of the preceding $\delta$ burst is larger than a threshold $d^{*}$ for VLP0-lesioned rats in $12 \mathrm{~h}$ dark periods. Durations are calculated using a window $w=4 \mathrm{~s}$ and a threshold $T h=1$ on the ratio $R_{\theta \delta}$. Insets show details of conditional probability distribution for short $d_{\delta_{i}}<0.2$ min. In all cases, the probability for longer $d_{\delta_{i}}$ systematically drops for increasing $d^{*}$ values (faster declining tail of the conditional distribution), whereas the probability for shorter $d_{\delta_{i}}$ significantly increases, as shown.

is a strong evidence for criticality underlying sleep micro-architecture; a signature of collective behavior over a range of time scales that emerges from neuronal interactions across brain areas. Such critical dynamics characterized by intermittent transitions between active and quiescent states at the integrated cortical level suggests the presence of a non-equilibrium mechanism that regulates sleep micro-architecture on time scales from seconds to an hour.

Our observations provide new insights about the role of the VLPO, and new methods to assess potential abnormal features in the sleep-wake cycle based on the analysis of cortical dynamics. Previous studies have demonstrated that lesions of the central cluster of the VLPO lead to a large decrease in delta power and NREM sleep time, and a fragmentation of the sleep-wake cycle (Lu et al., 2000; Vetrivelan et al., 2012). The results reported here indicate that, despite the decrease in delta power (Vetrivelan et al., 2012), VLPO lesions do not alter the dynamics of $\delta$-bursts. On the other hand, we found that lesions of VLPO neurons significantly affect the temporal organization of $\theta$-bursts. In particular, the power-law exponent characterizing $\theta$-bursts increases in rats with VLPO lesions, indicating a decreased likelihood for longlasting bursts and a higher probability to observe shorter bursts. This is an evidence of fragmentation of bursting activity in the $\theta$-wave band that parallels the increased fragmentation of sleep in VLPO-lesioned rats. Such deviations of the power-law exponent from the values measured in control rats indicate alteration in the optimal underlying mechanism for sleep regulation, and may be used as a marker of sleep disorders. Importantly, such changes in $\theta$-burst dynamics are indeed associated with a decreased anticorrelation between durations of consecutive $\theta$ - and $\delta$-bursts.
These findings, alongside with model simulations demonstrating that $\theta-\delta$ coupling is essential for the emerging scale-invariant temporal organization in these cortical rhythms, indicate that VLPO neurons may have dual role for both sleep and arousal/ brief wake activation.

The uncovered complex dynamics in $\theta$ and $\delta$ rhythms share striking similarities with natural non-equilibrium phenomena exhibiting self-organized criticality (Bak, 1996). In this context, bursts (active states) do not have a characteristic duration, and are separated by quiescent periods whose distribution depends on the details of the system and generally exhibit an exponential tail (Corral, 2004; Lombardi et al., 2014; Scarpetta and de Candia, 2014), and is an exponential for the paradigmatic sandpile model of self-organized criticality (Boffetta et al., 1999). Thus, in nonequilibrium systems exhibiting self-organized criticality, powerlaw and exponential behavior for active and quiet states coexist, and emerge out of the same regulatory mechanism. The duality of power-law and Weibull distribution in the bursting dynamics of $\theta$ and $\delta$ rhythms is closely reminiscent of this scenario, where scale-free $\theta$-bursts in cortical activity can be seen as avalanches or earthquakes (active states), whereas $\delta$-bursts can be interpreted as the quiet periods between active states. Following this analogy, we further demonstrated that the quiet times between consecutive $\theta$-burst above a given duration are described by a unique Gamma distribution, the scaling function that also characterizes earthquake dynamics (Corral, 2004).

The interpretation of $\theta$-bursts as active states and $\delta$-bursts as quiet states is consistent with the basic neurophysiological understanding of $\delta$ rhythm as the cortical default mode; indeed, lesion 
Data: durations of consecutive $\boldsymbol{\theta}$ - and $\boldsymbol{\delta}$-bursts

a

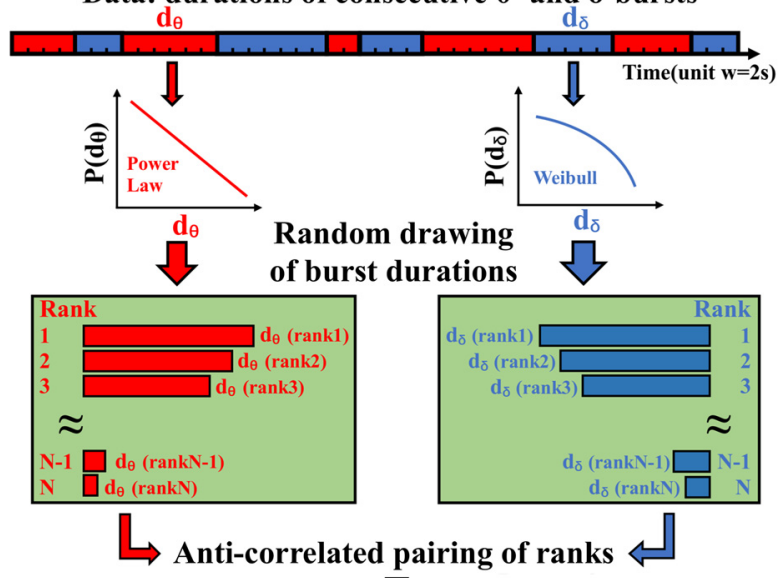

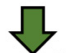

Model-generated $\boldsymbol{\theta}$ - and $\delta$-bursts time series

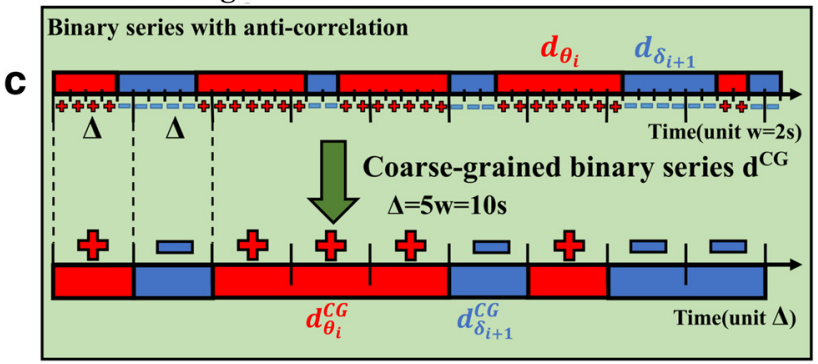

Figure 14. Schematic diagram of a phenomenological model to generate sequences of $\theta$ and $\delta$-burst durations with varied degree of anti-correlated coupling. $\boldsymbol{a}$, First, burst durations $d_{\theta}$ and $d_{\delta}$ are randomly drawn from the empirically obtained distributions (power-law and Weibull; Fig. 4) and separately ranked. Durations $d=n * w$ are a multiple of the scale of analysis (window size $w=2 \mathrm{~s}$ ). $\boldsymbol{b}$, Ranks of $\theta$ - and $\delta$-burst durations are then paired to form an anti-correlated sequence: if the rank $\left(d_{\theta}\right)$ of a $\theta$-burst is large, then the $\operatorname{rank}\left(d_{\delta}\right)$ of the following $d_{\delta}$-burst is selected to be smaller, and vice versa. Repeating this process leads to a sequence of generated $d_{\theta}$ and $d_{\delta}$ durations with a certain degree of anti-correlation. $c$, This newly generated anti-correlated time series is binarized, i.e., $+/-$ is assigned to each window $w$ that belongs either to a $d_{\theta}\left(\right.$ red, + ) or $d_{\delta}$ (blue, - ) duration, respectively. The binary time series is then coarse grained according to a majority rule applied over a window $\Delta=5 \mathrm{w}$. From the resulting CGBS, consecutive $\theta$ durations, $d_{\theta}^{C G}$, and $\delta$ durations, $d_{\delta}^{C G}$, are extracted.

and transection experiments have shown that interruption of sensory inputs to the cortex results in a cortical EEG similar to that in NREM sleep (von Economo, 1930; Bremer, 1935, 1937). In contrast, oscillations in the $\theta$ band are associated with REM state, arousals, and wakefulness (Boyce et al., 2016; Scammell et al., 2017). Because of the respective amount of wakefulness and REM sleep in our data (Vetrivelan et al., 2012), most of the analyzed $\theta$-bursts are likely associated with arousals and wake. Further, during wakefulness cortical $\theta$ waves are desynchronized with a wide range of burst durations, and correspondingly our analyses show that collective cortical activity follows robust scaling laws: power-law distribution for burst durations, long-range power-law correlations in the sequence of burst durations, and a scaling function describing quiet times between bursts.

All these features outline a general picture unifying previous empirical observations of spontaneous neuronal network dynamics at different levels, from networks of dissociated cortical neurons (Pasquale et al., 2008) and local field potentials in cortex slice cultures (Beggs and Plenz, 2003) and awake monkeys (Petermann et al., 2009), to the human brain (Linkenkaer-Hansen et al., 2001; Tagliazucchi et al., 2012; Palva et al., 2013; Shriki et al., 2013; Marinazzo et al., 2014), and the dynamics of sleep-stage
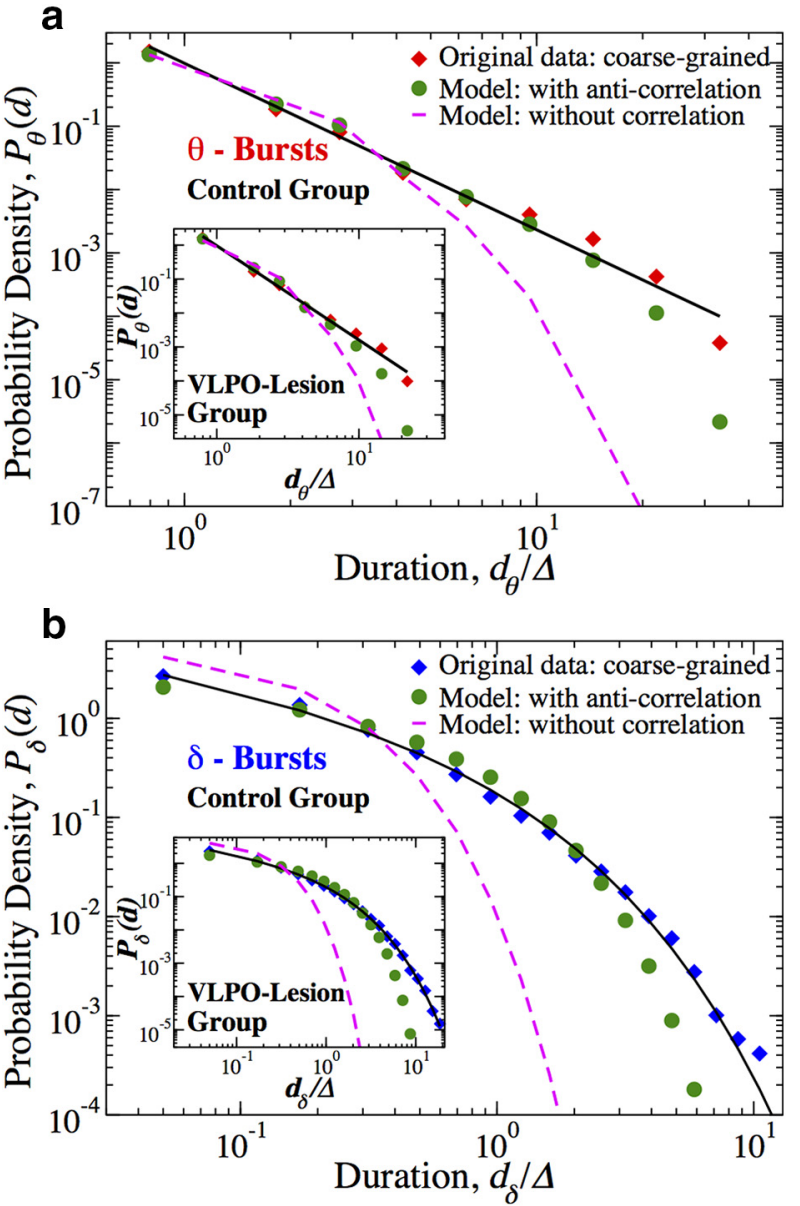

Figure 15. Anti-correlations between consecutive $\theta$ - and $\delta$-bursts durations are essential for emerging duality of power-law and Weibull dynamics. Probability distributions of $\theta$ - and $\delta$-burst durations from $24 \mathrm{~h}$ control and VLPO-lesioned rat data coarse-grained (CG) over a window $\Delta=10 \mathrm{~s}$, are compared with the distributions obtained from the model-generated coarse-grained binary time series of $\theta$ - and $\delta$-bursts durations with anti-correlations and without correlations (random pairing of $\theta$ - and $\delta$-bursts; Fig. 14). $\boldsymbol{a}$, Distributions $P_{\theta}(d)$ of $\theta$-burst durations for: (1) $24 \mathrm{~h}$ control rats data (red diamonds), (2) model-generated time series of $\theta$ and $\delta$-bursts durations with anti-correlations (green circles), and (3) model-generated time series with random pairing of $\theta$ - and $\delta$-bursts durations (magenta dashed line). Inset, Results from same analysis on $P_{\theta}(d)$ for the group of VLPO-lesioned rats. $\boldsymbol{b}$, Distribution $P_{\delta}(d)$ of $\delta$-burst durations for: (1) $24 \mathrm{~h}$ control rats data (blue diamonds), (2) model-generated time series with anti-correlations (green circles), and (3) model-generated time series with random pairing of $\theta$-and $\delta$-bursts durations (magenta dashed line). Inset, Results from same analysis on $P_{\delta}(d)$ for the group of VLPO-lesioned rats. In both $\boldsymbol{a}$ and $\boldsymbol{b}$, durations are in units of $\Delta$, which is the window size used to coarse grain the sequences of $\theta$-and $\delta$-bursts durations. The distributions obtained from the model using anti-correlated $d_{\theta}$ and $d_{\delta}$ pairing (green circles) closely match the duration distributions for the original data (diamonds), power-law for $P_{\theta}(d)$ and Weibull for $P_{\delta}(d)$, for both control and VLPO-lesioned rats. In contrast, a random pairing of $d_{\theta}$ and $d_{\delta}$ produces duration distributions following the Poisson functional form (magenta dashed lines) that significantly deviates from the original data.

and arousal transitions across species (Lo et al., 2002, 2004, 2013; Blumberg et al., 2005; Sorribes et al., 2013), where either distributions or temporal correlations of active events have been studied and discussed in the context of self-organized criticality. Crucially, here we demonstrated presence of the full spectrum of scaling characteristics typical for systems self-organizing at criticality. Furthermore, we linked our observations to the collective behavior of a key sleep-promoting neuronal population leading to emerging cortical rhythms in relation to physiological alternation of sleep and wake. We found that the power-law scaling exponent of the distribution for $\theta$-burst durations in control rats 
is $\alpha \simeq 2.4$, close to the power-law exponent for the distribution of neuronal avalanches durations (Beggs and Plenz, 2003; Pasquale et al., 2008), and to $\alpha \simeq 2.2$ reported for arousal/wake episodes in coarse-grained sleep-stage recordings in humans (Lo et al., 2002) and other species (Lo et al., 2004; Blumberg et al., 2005; Sorribes et al., 2013; Dvir et al., 2018). Importantly, we observed that lesions of VLPO lead to significantly higher values for the $\theta$-burst power-law exponent, an effect that may be interpreted as a transition into a sub-critical behavior (a deviation from emergent criticality due to alteration of the underlying control mechanism)(Lombardi et al., 2012, 2017; Poil et al., 2012).

In summary, the reported duality of power-law and Weibull distribution and the scaling features for a full spectrum of dynamic characteristics in the bursting activity of $\theta$ and $\delta$ rhythms strongly support the hypothesis of an underlying critical dynamics for sleep regulation. These evidences lay the foundation of a new paradigm for the investigation of sleep dynamics and sleepstage transitions mechanisms, considering sleep micro-architecture as result of a non-equilibrium process and self-organization among neuronal assemblies to maintain a critical state (a behavior that is in stark contrast to the homeostasis paradigm of sleep regulation at large time scales). Within the criticality framework arousals/brief wake and sleep-stage dynamics emerge out of a common regulatory mechanism, where arousals play an essential part in maintaining a non-equilibrium behavior at criticality, as suggested by our observations of coupling between consecutive $\theta$ - and $\delta$-bursts durations. Systems at criticality exhibit high susceptibility and sensitivity to interactions, leading to cooperative behaviors over a range of space/time scales, and thus maintaining a critical state is important for system's flexibility and for generating spontaneous transitions (Ansmann et al., 2016; Lehnertz et al., 2018). Such transitions cannot occur intrinsically in a homeostatic (equilibrium) system. In the context of sleep micro-architecture, the proposed criticality-based paradigm may provide new insights on the origin and mechanisms underlying the dynamics of sleep-stage and arousal transitions, and offers a unifying picture of sleep and wake.

\section{References}

Achermann P, Borbély AA (2003) Mathematical models of sleep regulation. Front Biosci 8:S683-S693.

Amaral DG, Price JL (1983) An air pressure system for the injection of tracer substances into the brain. J Neurosci Methods 9:35-43.

Ansmann G, Lehnertz K, Feudel U (2016) Self-induced switchings between multiple space-time patterns on complex networks of excitable units. Phys Rev X 6:011030.

Bak P (1996) How nature works. New York: Copernicus.

Beggs JM, Plenz D (2003) Neuronal avalanches in neocortical circuits. J Neurosci 23:11167-11177.

Behn CG, Brown EN, Scammell TE, Kopell NJ (2007) Mathematical model of network dynamics governing mouse sleep-wake behavior. J Neurophysiol 97:3828-3840.

Blumberg MS, Seelke AM, Lowen SB, Karlsson KA (2005) Dynamics of sleep-wake cyclicity in developing rats. Proc Natl Acad Sci U S A 102: $14860-14864$.

Boffetta G, Carbone V, Giuliani P, Veltri P, Vulpiani A (1999) Power laws in solar flares: self-organized criticality or turbulence? Phys Rev Lett 83: 4662.

Borbély AA, Achermann P (1999) Sleep homeostasis and models of sleep regulation. J Biol Rhythms 14:559-570.

Boyce R, Glasgow SD, Williams S, Adamantidis A (2016) Causal evidence for the role of rem sleep theta rhythm in contextual memory consolidation. Science 352:812-816.

Bremer F (1935) Cerveau "Isole" et physiologie du sommeil. Comptes rendus de la Societe de Biologie 118:1235-1241.

Bremer F (1937) L' activite cerebrale au cours du sommeil et de la narcose. Contribution a l'etude mecanistique du sommeil. Academie royale de medecine de Belgique 2:68-86.
Brown RE, Basheer R, McKenna JT, Strecker RE, McCarley RW (2012) Control of sleep and wakefulness. Physiol Rev 92:1087-1187.

Chen Z, Ivanov PC, Hu K, Stanley HE (2002) Effect of nonstationarities on detrended fluctuation analysis. Phys Rev E Stat Nonlin Soft Matter Phys 65:041107.

Chen Z, Hu K, Carpena P, Bernaola-Galvan P, Stanley HE, Ivanov PC (2005) Effect of nonlinear filters on detrended fluctuation analysis. Phys Rev E Stat Nonlin Soft Matter Phys 71:011104.

Chialvo D (2010) Emergent complex neural dynamics. Nat Physics 6:744-750.

Corral Á (2004) Long-term clustering, scaling, and universality in the temporal occurrence of earthquakes. Phys Rev Lett 92:108501.

Corral Á (2006) Dependence of earthquake recurrence times and independence of magnitudes on seismicity history. Tectonophysics 424:177-193.

Daley DJ, Vere-Jones D (1988) An introduction to the theory of point processes. New York: Springer.

de Arcangelis L, Godano C, Grasso JR, Lippiello E (2016) Statistical physics approach to earthquake occurrence and forecasting. Phys Rep 628:1-91.

Dvir H, Elbaz I, Havlin S, Appelbaum L, Ivanov PC, Bartsch RP (2018) Neuronal noise as an origin of sleep arousals and its role in sudden infant death syndrome. Sci Adv 4:eaar6277.

Franken P, Dijk DJ, Tobler I, Borbély AA (1991) Sleep deprivation in rats: effects on EEG power spectra, vigilance states, and cortical temperature. Am J Physiol 261:R198-R208.

Goldberger AL, Amaral LA, Hausdorff JM, Ivanov PCh, Peng CK, Stanley HE (2002) Fractal dynamics in physiology: alterations with disease and aging. Proc Natl Acad Sci U S A 99:2466-2472.

Halász P (1998) Hierarchy of micro-arousals and the microstructure of sleep. Neurophysiol Clin 28:461-475.

Hirshkowitz M (2002) Arousals and anti-arousals. Sleep Med 3:203-204.

Hu K, Ivanov PC, Chen Z, Carpena P, Stanley HE (2001) Effect of trends on detrended fluctuation analysis. Phys Rev E Stat Nonlin Soft Matter Phys 64:011114.

Hu K, Ivanov PC, Hilton MF, Chen Z, Ayers RT, Stanley HE, Shea SA (2004) Endogenous circadian rhythm in an index of cardiac vulnerability independent of changes in behavior. Proc Natl Acad Sci U S A 101:1822318227.

Ivanov PC (2007) Scale-invariant aspects of cardiac dynamics across sleep stages and circadian phases. IEEE Eng Med Biol Mag 26:33-37.

Ivanov PC, Rosenblum MG, Peng CK, Mietus J, Havlin S, Stanley HE, Goldberger AL (1996) Scaling behaviour of heartbeat intervals obtained by wavelet-based time-series analysis. Nature 383:323-327.

Ivanov PC, Amaral LA, Goldberger AL, Havlin S, Rosenblum MG, Struzik ZR, Stanley HE (1999) Multifractality in human heartbeat dynamics. Nature 399:461-465.

Kantelhardt J, Havlin S, Ivanov PC (2003) Modeling transient correlations in heartbeat dynamics during sleep. Europhys Lett 62:147.

Kopell N, Ermentrout GB, Whittington MA, Traub RD (2000) Gamma rhythms and beta rhythms have different synchronization properties. Proc Natl Acad Sci U S A 97:1867-1872.

Lehnertz K, Zabawa L, Tabar MRR (2018) Characterizing abrupt transitions in stochastic dynamics. New J Physics 20:113043.

Linkenkaer-Hansen K, Nikouline VV, Palva JM, IImoniemi RJ (2001) Long-range temporal correlations and scaling behavior in human brain oscillations. J Neurosci 21:1370-1377.

Liu KK, Bartsch RP, Lin A, Mantegna RN, Ivanov PC (2015) Plasticity of brain wave network interactions and evolution across physiologic states. Front Neural Circuits 9:62.

Lombardi F, Herrmann HJ, Perrone-Capano C, Plenz D, de Arcangelis L (2012) Balance between excitation and inhibition controls the temporal organization of neuronal avalanches. Phys Rev Lett 108:228703.

Lombardi F, Herrmann HJ, Plenz D, de Arcangelis L (2014) On the temporal organization of neuronal avalanches. Front Syst Neurosci 8:204.

Lombardi F, Herrmann HJ, de Arcangelis L (2017) Balance between excitation and inhibition determines $1 /$ f power spectrum in neuronal networks. Chaos 27:047402.

Lo CC, Amaral LN, Havlin S, Ivanov PC, Penzel T, Peter JH, Stanley HE (2002) Dynamics of sleep-wake transitions during sleep. Europhys Lett 57:625.

Lo CC, Chou T, Penzel T, Scammell TE, Strecker RE, Stanley HE, Ivanov PC (2004) Common scale-invariant patterns of sleep-wake transitions across mammalian species. Proc Natl Acad Sci U S A 101:17545-17548. 
Lo CC, Bartsch RP, Ivanov PC (2013) Asymmetry and basic pathways in sleep-stage transitions. Europhys Lett 102:10008.

Lu J, Greco MA, Shiromani P, Saper CB (2000) Effect of lesions of the ventrolateral preoptic nucleus on NREM and REM sleep. J Neurosci 20:3830-3842.

Lu J, Bjorkum AA, Xu M, Gaus SE, Shiromani PJ, Saper CB (2002) Selective activation of the extended ventrolateral preoptic nucleus during rapid eye movement sleep. J Neurosci 22:4568-4576.

Marinazzo D, Pellicoro M, Wu G, Angelini L, Cortés JM, Stramaglia S (2014) Information transfer and criticality in the Ising model on the human connectome. PLoS One 9:e93616.

Munoz MA (2018) Colloqium: criticality and dynamical scaling in living systems. Rev Mod Phys 90:031001.

Olbrich E, Achermann P, Wennekers T (2011) The sleeping brain as a complex system. Philos Trans A Math Phys Eng Sci 369:3697-3707.

Paczuski M, Boettcher S, Baiesi M (2005) Interoccurrence times in the BakTang-Wiesenfeld Sandpile model: a comparison with the observed statistics of solar flares. Phys Rev Lett 95:181102.

Palva JM, Zhigalov A, Hirvonen J, Korhonen O, Linkenkaer-Hansen K, Palva S (2013) Neuronal long-range temporal correlations and avalanche dynamics are correlated with behavioral scaling laws. Proc Natl Acad Sci U S A 110:3585-3590.

Pasquale V, Massobrio P, Bologna LL, Chiappalone M, Martinoia S (2008) Self-organization and neuronal avalanches in networks of dissociated cortical neurons. Neuroscience 153:1354-1369.

Paxinos G, Watson C (2004) The rat brain in stereotaxic coordinates. 5 ed. Amsterdam; Boston: Elsevier Academic Press.

Peng CK, Buldyrev SV, Havlin S, Simons M, Stanley HE, Goldberger AL (1994) Mosaic organization of DNA nucleotides. Phys Rev E Stat Phys Plasmas Fluids Relat Interdiscip Topics 49:1685-1689.

Petermann T, Thiagarajan TC, Lebedev MA, Nicolelis MA, Chialvo DR, Plenz D (2009) Spontaneous cortical activity in awake monkeys composed of neuronal avalanches. Proc Natl Acad Sci U S A 106:15921-15926.

Poil SS, Hardstone R, Mansvelder HD, Linkenkaer-Hansen K (2012) Criticalstate dynamics of avalanches and oscillations jointly emerge from balanced excitation/inhibition in neuronal networks. J Neurosci 32:9817-9823.

Pruessner G (2012) Self-organised criticality: theory, models and characterisation. Cambridge UK: Cambridge UP.

Ribeiro TL, Copelli M, Caixeta F, Belchior H, Chialvo DR, Nicolelis MA, Ribeiro S (2010) Spike avalanches exhibit universal dynamics across the sleep-wake cycle. PLoS One 5:e14129.

Rodriguez AV, Funk CM, Vyazovskiy VV, Nir Y, Tononi G, Cirelli C (2016) Slow-wave activity increase after extended wake? Assessing the effects of increased cortical firing during wake and sleep. J Neurosci 36:12436-12447.

Sakurai T (2007) The neural circuit of orexin (hypocretin): maintaining sleep and wakefulness. Nat Rev Neurosci 8:171-181.

Saper CB, Chou TC, Scammell TE (2001) The sleep switch: hypothalamic control of sleep and wakefulness. Trends Neurosci 24:726-731.
Saper CB, Cano G, Scammell TE (2005) Homeostatic, circadian, and emotional regulation of sleep. J Comp Neurol 493:92-98.

Saper CB, Fuller PM, Pedersen NP, Lu J, Scammell TE (2010) Sleep state switching. Neuron 68:1023-1042.

Scammell TE, Arrigoni E, Lipton JO (2017) Neural circuitry of wakefulness and sleep. Neuron 93:747-765.

Scarpetta S, de Candia A (2014) Alternation of up and down states at a dynamical phase-transition of a neural network with spatiotemporal attractors. Front Syst Neurosci 8:88.

Schmitt DT, Stein PK, Ivanov PC (2009) Stratification pattern of static and scale-invariant dynamic measures of heartbeat fluctuations across sleep stages in young and elderly. IEEE Trans Biomed Eng 56:1564-1573.

Sherin JE, Shiromani PJ, McCarley RW, Saper CB (1996) Activation of ventrolateral preoptic neurons during sleep. Science 271:216-219.

Shriki O, Alstott J, Carver F, Holroyd T, Hanson RN, Smith ML, Coppola R, Bullmore E, Plenz D (2013) Neuronal avalanches in the resting meg of the human brain. J Neurosci 33:7079-7090.

Sorribes A, Thornorsteinsson H, Porsteinsson H, Arnardóttir H, Jóhannesdóttir IP, Sigurgeirsson B, De Polavieja GG, Karlsson KÆ (2013) The ontogeny of sleep-wake cycles in zebrafish: a comparison to humans. Front Neural Circuits 7:178.

Stacy EW (1962) A generalization of the gamma distribution. Ann Math Stat 33:1187-1192.

Stanley H (1987) Introduction to phase transitions and critical phenomena. New York: Oxford UP.

Steriade M, Nuñez A, Amzica F (1993) A novel slow ( $<1 \mathrm{~Hz}$ ) oscillation of neocortical neurons in vivo: depolarizing and hyperpolarizing components. J Neurosci 13:3252-3265.

Tagliazucchi E, Balenzuela P, Fraiman D, Chialvo DR (2012) Criticality in large-scale brain fMRI dynamics unveiled by a novel point process analysis. Front Physiol 3:15.

Taqqu MS, Teverovsky V, Willinger W (1995) Estimators for long-range dependence: an empirical study. Fractals 3:785-798.

Tobler I, Borbély AA (1986) Sleep eeg in the rat as a function of prior waking. Electroencephalogr Clin Neurophysiol 64:74-76.

Trachsel L, Tobler I, Achermann P, Borbély AA (1991) Sleep continuity and the rem-nonREM cycle in the rat under baseline conditions and after sleep deprivation. Physiol Behav 49:575-580.

Vetrivelan R, Fuller PM, Yokota S, Lu J, Saper CB (2012) Metabolic effects of chronic sleep restriction in rats. Sleep 35:1511-1520.

von Economo C (1930) Sleep as a problem of localization. J Nerv Ment Dis $71: 1-5$.

Welch P (1967) The use of fast Fourier transform for the estimation of power spectra: a method based on time averaging over short, modified periodograms. IEEE Trans Audio Electroacoust 15:70-73.

Xu L, Ivanov PC, Hu K, Chen Z, Carbone A, Stanley HE (2005) Quantifying signals with power-law correlations: a comparative study of detrended fluctuation analysis and detrended moving average techniques. Phys Rev E Stat Nonlin Soft Matter Phys 71:051101. 